\title{
Experimental Investigation of Pebble Flow Dynamics using Radioactive Particle Tracking Technique in a Scaled-down Pebble Bed Modular Reactor (PBMR)
}

\author{
Vaibhav Khane ${ }^{[1]}$, I. A. Said ${ }^{[1]}$, and Muthanna H. Al-Dahhan ${ }^{[1]^{*}}$ \\ ${ }^{[1]}$ Department of Chemical and Biochemical Engineering, Missouri University of Science and \\ Technology, 110 Bertelsmeyer Hall, 1101 N. State Street, Rolla, MO, United States \\ * Corresponding Author (aldahhanm@mst.edu)
}

\begin{abstract}
The Pebble Bed Modular Reactor (PBMR) is a type of very-high-temperature reactor (VHTR) that is conceptually very similar to moving bed reactors used in the chemical and petrochemical industries. In a PBMR core, nuclear fuel is in the form of pebbles and moves slowly under the influence of gravity. In this work, an integrated experimental and computational study of granular flow in a scaled-down cold flow PBMR was performed. A continuous pebble recirculation experimental set-up, mimicking the flow of pebbles in a PBMR was designed and developed. An experimental investigation of pebble flow dynamics in a scaled down test reactor was carried out using a non-invasive radioactive particle tracking (RPT) technique that used a cobalt-60 based tracer to mimic pebbles in terms of shape, size and density. A cross-correlation based position reconstruction algorithm and RPT calibration data were used to obtain results about Lagrangian trajectories, the velocity field, and residence time distributions. The RPT technique results a serve as a benchmark data for assessing contact force models used in the discrete element method (DEM) simulations.
\end{abstract}

\section{Key Words}

Pebble Bed Modular Reactor (PBMR); Granular Flow; Radioactive Particle Tracking (RPT); Pebbles Flow Dynamics; Discrete Element Method (DEM). 


\section{Introduction}

The VHTR is one of the fourth generation nuclear reactors among six designs and uses gaseous coolant. The VHTR is either a Prismatic Block Reactor (PBR) or Pebble Bed Modular Reactor (PBMR). Both these VHTR designs contain their fuel in the form of TRISO (Tri-isotropic) nuclear fuel particles[1]. In a PBMR, nuclear fuel is in the form of spherical pebbles and move slowly in the core under the influence of gravity. The fuel pebbles are continuously re-circulated through the core and are monitored for burn-up. This continuous re-circulation feature eliminates the need to shut down the rector for refueling. The helium gas is used as a coolant to remove the heat from the fuel and it flows through a complex interconnected network of voids formed between pebbles. The flow of pebbles under gravity in a pebble bed reactor is a slow and dense type granular flow. The moving core of a PBMR is a cause of concern from a nuclear safety and performance evaluation point of view, which demands a basic understanding of the physics governing the dense granular flow.

\section{Previous work}

An experimental study was performed at Australian Atomic Energy Commission [2] to track pebble trajectories at pre-defined intervals of time using a collimated detector based radioactive particle tracking (RPT) technique from the outside in recirculated randomly packed beds under different operating conditions and bed parameters. The results showed that the trajectories of pebbles were in a straight line and there was little interference or crossing between pebble trajectories. These results were analyzed to identify the boundaries of four different flow zones typically observed during the discharge of granular materials[3]. The version of the RPT technique that was used in this study has some inherent limitations: upper limit on tracking speed due to dynamically moving platform and lower counts were recorded due to collimated detectors [4]. Kadak and Bazant [5] investigated a scaled-down pebble bed modular reactor (PBMR) for 
movement of fuel and graphite pebbles in a bi-disperse core concept to answer if they move in a streamlined manner or in a random haphazard fashion under different experimental models:

$1.180^{\circ}$ half-model 2. 3-D opaque cylinder. In a $180^{\circ}$ half-model and continuous flow experimental set-up with dynamic central column, visual tracking of pebbles at the mid-plane transparent wall was carried out. $180^{\circ}$ half-model suffers from a 'wall effect' which alters the overall flow behavior. A study in a full three-dimensional opaque cylinder was also carried out to overcome this 'wall effect'. The effect of different bottom cone angles and exit opening diameters was studied as a part of the study carried out at M.I.T [5]. The experimental set-up used in this study at M.I.T has some limitations: there was no continuous and automatic recirculation of exiting pebbles. Tsinghua University in China designed and developed a 10 MWth prototype pebble bed reactor which is called HTR-10 and the characteristics of pebble flow dynamics were investigated experimentally under different cone angles and different surface roughnesses of pebbles in a two dimensional 1:5 scaled down model of a pebble bed core $[6,7]$. A stable two-region arrangement of the core was established and maintained during experiments. Yang et al.[6] observed a stagnant zones in the corner of the experimental set-up. In addition, the motion of pebbles in the pebble bed was reported to be of collective type and intermittent. Pebbles motion paths were reported to be of streamline form. Also, investigations in taller experimental vessel were performed to verify the feasibility of two-region design [8]. Additionally, a discrete element method (DEM) simulations based study was carried out at Tsinghua University to verify the experimental observations and simulated effect of different cone angles on pebble motion. The main limitation of the experimental work carried out at Tsinghua University was the use of a two dimensional experimental set-up instead of the actual three dimensional geometry. At North Carolina State University (NCSU), a study of the granular 
flow in a PBMR was carried out using collimated detectors based radioactive particle tracking technique similar to the tracker used in Gatt's study [4]. The main aim was to explore the technique's potential and its limitations through some error and sensitivity analysis. In another continuation work at NCSU [9], a dual measurement system for tracking flow of pebbles in a PBMR was developed. A comparison of results obtained using collimated and non-collimated detectors based RPT technique at NCSU with Gatt's study suggested agreement on trajectories results. This study at NCSU did not involve continuous pebbles recirculation experimental setup. In another work at Beijing Forestry University in China [6], combined DEM and experimental study of flow of pebbles was carried out in a transparent semi-cylindrical silo. The main limitations of this combined DEM and experimental study were the use of semi-cylindrical geometry and the use of a visual tracking technique. It is clear from the review of previous experimental studies and measurement methods that there was a need to carry out a threedimensional pebble flow dynamics study in a continuous recirculation experimental set-up using advanced flow visualization techniques. Hence, in this work continuous pebbles recirculation experimental set-up mimicking 3-D flow of pebbles in a PBMR was developed and an advanced and non-invasive radioactive particle tracking (RPT) technique was used to gather information about the pebble velocity field. The RPT technique has no limitation on operating conditions, opacity, and system design and configurations and hence is suitable for the study of dense and granular flow. An investigation of the pebble flow field is of paramount importance from reactor neutronics and coolant thermal hydraulics point of view [10]. The obtained engineering knowledge and data can be used in the validation of computational methods which are/will be ultimately used to evaluate pebble flow and structural characterization in actual scale geometry. 
Also, such knowledge and data will be valuable for the proper design, scale-up and commercial demonstration of pebble bed reactor technology.

\section{Current Work}

\subsection{Experimental Setup}

A cold flow three dimensional continuous pebble recirculation experimental set-up was designed, developed and tested (Figure 1) at Missouri S\&T. It has unique features of i) adjustable flow of pebbles at the bottom of the pebble bed without jamming by developing a unique mechanical design that does not affect the dynamic flow of pebbles in the bed, ii) the exit pebbles are recirculated back using a continuously moving conveyor, and iii) the pebbles return at various locations at the top of the bed in a non-violent manner by developing a unique design of the returning tubing and connections that slow down the dropping pebbles to be placed at a defined and desirable location of the top cross-section of the bed in a non-violent manner. This design and its features can be applied to any size of the studied pebbles and bed diameter. In this work The pebble bed test reactor made of acrylic (1foot outer diameter with 11.95 inch inside diameter and 1 foot in height) filled with glass marbles of $1 / 2$ " diameter having density $\sim 2.5 \mathrm{~g} / \mathrm{cm} 3$ was used. $1 / 2$ " diameter glass marbles are found suitable to start with, from tracer preparation point of view and representing actual pebbles flow (actual density of pebbles $\sim 1.8$ $\mathrm{g} / \mathrm{cm} 3$ ). The diameter of test reactor is selected based on considerations of representing the wall effect as the nature of packing will affect the subsequent flow of pebbles. A large diameter test reactor will have significant attenuation demanding a stronger radioactive source and hence was avoided in this work. A one foot diameter of test reactor will yield a diameter aspect ratio (which is defined as a ratio of inside cylinder diameter to the pebble diameter) of 23.9. Such an aspect ratio is capable of introducing a wall-effect induced oscillatory variation in radial porosity observed in an actual reactor. An exit flow rate of one pebble every five seconds was used in all 
experiments. The inlet control mechanism was connected to a top plate (diameter matching with the reactor) with 17 holes. These holes were provided to return the pebble at 17 different radial positions. The vertical leg of the conveyor belt was kept at a sufficient distance $(\sim 150 \mathrm{~cm})$ away from the test reactor. This allowed ease in implementing the of advanced flow visualization techniques around the test reactor. Further details about the exit mechanism design and development and the inlet control system can be found elsewhere[11].

\subsection{Implementation of RPT Technique on continuous recirculation experimental set-up.}

The radioactive particle tracking (RPT) technique, a non-invasive radioisotope based flow visualization technique was implemented on the continuous cold-flow pebble recirculation experimental set-up at Missouri S\&T. The RPT technique is capable of providing information about the occurrence of three-dimensional pebbles, Lagrangian trajectory, velocity and its components, overall and local residence time distribution, stagnant zones, and other related solids flow dynamic parameters in a non-invasive manner. In general, the RPT technique uses a single or multiple radioactive particles (also called as a tracer) emitting $\gamma$-rays (i.e. radiotracer particle) whose motion is followed in the 3-D domain of the whole system using either collimated or non-collimated scintillation detectors[12-14]. In the RPT technique used in this work (Figure 2), the instantaneous tracer position was identified by simultaneously monitoring

photo-peak counts received by a set of non-collimated sodium iodide (NaI) scintillation detectors which were arranged strategically around the system [15-18]. The counts received in each detector are a function of the distance between the detector and the particle, and the attenuating material inventory present between the tracer and the detector. The forward problem of finding the instantaneous position of particle based on intensities received at the detectors is solved by performing calibration experiments that could be integrated with computation, using Monte 
Carlo simulation approach or mechanistic correlations. RPT calibration experiments, which are performed prior to actual RPT experiments, are carried out at the same operating conditions as that of the actual experiment to mimic the radiation attenuation in the system. The radioactive tracer (Co-60 used in this work) is placed at various known locations and the counts received at each detector are recorded. Using this information calibration curves, which are essentially distance-count map for each detector, are established. The instantaneous position of the tracer can be discovered with the help of various in-house developed position reconstruction algorithms $[12,13,16,18-25]$ and calibration curves. Using this instantaneous position data, Lagrangian trajectories, instantaneous and time averaged velocities and their component fields and various turbulent parameters, if applicable (Reynolds stresses, turbulent kinetic energy, turbulent eddy diffusivities, etc.) can be determined. Figure 2 shows the implementation of the RPT technique around this continuous pebble recirculation set-up. It is noteworthy that RPT is the only noninvasive and quantitative measurement technique capable of providing a full description of the 3-D flow field in highly opaque reactors and can provide particle Lagrangian velocities throughout the domain. The high penetration capability of gamma rays makes the RPT technique suitable for visualization of flow through dense and opaque multiphase systems. This is one of the main advantages of the RPT technique over optics based flow visualization techniques. Among different velocimetry techniques available, the technique of RPT is not only accurate but also provides data in a non-invasive manner without any limitations from system opacity. This avoids the introduction of an intrusive probe which affects the flow dynamics. Hence, it is possible to capture the true multiphase flow dynamics with the RPT technique. 


\subsubsection{Radioactive Tracer Source Selection and Preparation}

A feasibility study was carried out using Scandium-46 and cobalt-60 based tracer particle. A cobalt-60 based tracer particle was used due to its prolonged half-life $(\sim 5.2$ years) as compared to the shorter half-life of scandium-46 (half-life $\sim 84$ days), its higher gamma-ray energies and its lesser irradiation time requirement in nuclear reactor as a part of the tracer preparation. Cobalt-60 was found to be suitable for this study of granular flow as the movement of pebbles was very slow and did not require any half- life correction unlike scandium-46. A cobalt particle with a diameter of $600 \mu \mathrm{m}$ was selected for irradiation with neutrons in Missouri University Research Reactor (MURR) to optimize irradiation time in the nuclear reactor. A hole with a $1 \mathrm{~mm}$ diameter and $\sim 7 \mathbf{m m}$ length was drilled in a Teflon particle to accommodate this $600 \mu \mathrm{m}$ radioactive cobalt particle. The density of the composite tracer particle was then matched with that of glass marbles by matching their masses. The mass of this composite Teflon particle with a dummy cobalt particle and steel screw cap was matched with average mass of $1 / 2$ " glass marbles by selecting a suitable length for the screw cap and adjusting the air gap. A screw cap made from steel (1 mm diameter and $3.2 \mathrm{~mm}$ in length) matched the mass of the composite tracer particle (2.48 grams) with the average mass of glass marbles (2.49 grams). Figure 3 shows a schematic diagram and actual picture of the RPT tracer particle used in this PBMR study.

\subsubsection{RPT Detector Arrangement}

In this work, sixteen non-collimated sodium iodide (NaI) scintillation detectors were used. Roy et al. [26] recommended a symmetric distribution of scintillation detectors around the system and an alternate staggering of adjacent plane detectors during RPT technique implementation. Also, Roy et al. suggested that better resolution and good sensitivity in position reconstruction can be achieved using four detectors per plane. Hence, 
the number of detectors per plane was 4 (at $90^{\circ}$ to in-plane neighboring detectors). The adjacent plane detectors were staggered at $45^{\circ}$ in order to improve the accuracy and resolution [26]. 3 "' $(0.0762 \mathrm{~m})$ of distance was maintained between two neighboring in-plane arrangements of detectors. Also, 3" (0.0762 $\mathrm{m})$ of gap was maintained between the front face of the detectors and the wall of the test reactor. Table 1 shows the position coordinates of the RPT detectors. Figure 4 shows the arrangement of the detectors around an experimental set-up.

\subsubsection{RPT Calibration}

An analytical solution to the forward problem of RPT, i.e. finding the instantaneous tracer position based upon counts data received in the detectors, is not impossible. This problem was solved by performing a set of calibration experiments at the same operating conditions as those of the actual RPT experiments. A calibration methodology involving a manual calibration apparatus (Figure 5) was used for this work. The manual calibration apparatus consists of a rod that has a vial at its tip to contain a radioactive particle. With the help of a guide bush arrangement the rod can slide into/out of place with the radioactive particle at any desired height of the test reactor. The position can be recorded with the help of a scale attached to the rod. This rod can be placed at selected radial positions with the help of threaded holes in the top plate. The prepared tracer particle (cobalt-60) was positioned at different radial $(0 \mathrm{~cm}, 7.62 \mathrm{~cm}$ and 13.97 $\mathrm{cm})$ and azimuthal positions $\left(0^{\circ}\right.$ through $360^{\circ}$ in steps of $\left.45^{\circ}\right)$ at different vertical heights (incremental steps of $2 \mathrm{~cm}$ ) using a manual calibration apparatus (Figure 5 ) and photo-peak counts were recorded in each detector (16 detectors surrounding the system) for each tracer position. A total 376 calibration positions were used to carry out the RPT calibration. This data was used to generate calibration maps relating counts with position, which are helpful in the position reconstruction step. A sampling frequency of $6 \mathrm{~Hz}$ was chosen because of the slow movement of the pebbles. Calibration curves (Figure 6) generated for each detector indicated that 
there is a spread in count readings for the same tracer-detector distance. This suggests that counts received at the detectors are not only a function of the tracer-detector distance but also of the attenuation characteristics of medium in between the tracer and the detector. This spread in counts also known as 'band effect' is due to highly un-isotropic attenuating medium. The Solid angle subtended at the tracer by the detector plays an important role towards this 'band effect'. At larger tracer-detector distances, the effect of the solid angle diminishes and less spread in counts is observed. At smaller tracer-detector distances, the effect of the solid angle is dominant and hence, a broader spread in recorded counts is observed. This spread of counts for the same distance of tracer from the detector posed additional challenges in particle location reconstruction. Hence, a cross-correlation based position reconstruction algorithm, which takes into account the geometry as well as the attenuating medium effects, was used in this PBMR study [23]. This methodology can also be used to conduct a zone search using the zone surrounding the detectors which helps minimize the effects of counts spreading on reconstructing the tracer particle locations. This enhances the accuracy of the position reconstruction as outlined in the following section.

\subsubsection{RPT experiments}

During RPT experiments, the radioactive tracer particle (cobalt-60) was seeded at different radial positions using a seeding tube and was allowed to move freely with the rest of the pebbles (glass marbles), while the non-collimated sodium iodide (NaI) scintillation detectors (sixteen NaI detectors) kept collecting counts continuously at a frequency of $6 \mathrm{~Hz}$ until they left the system from the bottom opening. A sampling frequency of $6 \mathrm{~Hz}$ was chosen because of the very slow movement of the pebbles. It was the smallest sampling frequency possible with the new DAQ system of RPT. At smaller sampling frequencies, the signal-to-noise ratio was 
better due to the larger sampling time. The experiment was conducted as a once-through flow system where the particle movement was very slow. Hence, it is not practical time wise and from RPT calibration point of view due to the reduction in the radioactivity of the particle with time to let the radioactive particle visits each location many times. Therefore, the concept of achieving large number of occurrences in each location to ensure proper statistics is not applicable in this experiments. Therefore, in this work we have presented the data of the tracer particle as a once through movement to demonstrate the lagrangian trajectory of the pebbles at various radial locations inside the bed. This presentation is the proper one because the accuracy of reconstructing the location of the tracer particle inside the bed is acceptable.

\subsubsection{RPT Reconstruction Algorithm}

The main aim of the RPT position reconstruction algorithm is to find the instantaneous position of the tracer particle based on the counts recorded in a set of detectors with least possible reconstruction error. There are four main types of RPT position reconstruction algorithms $[21,27-30]$ which are as follows.

- Weighted Least Square Regression Method

- Monte Carlo Method

- Feed Forward Neural Network Method

- Cross-Correlation Based Method involving Semi-Empirical Model

The weighted least square regression method has poor accuracy and resolution in dense flows [20]. This is due to the basic assumption that counts recorded depend only on the particle-detector distance and are independent of the geometry of the system, 
attenuating medium, etc. The monte Carlo method $[28,31]$ accounts for the effect of the geometry, solid angle, and characteristics of an attenuating medium. The modeling of an attenuating medium in between the tracer and the detector is usually carried out using the holdup distribution profile. However, the effect of flow conditions on the attenuating medium is either taken as a constant [32] or estimated using a time-averaged holdup profile [28]. The change in the intensity of counts with changes in the holdup distributions is large. Using the constant holdup value or time-averaged hold-up profile, where its constants are estimated by regression, introduces errors into the computationally expensive and sophisticated Monte Carlo based model. This is one of the main drawbacks of this method. The feed forward neural network method [33] uses a black-box model employing neural network. The main drawback of this method is that the model used does not have any physical significance and employs a huge number of fitting parameters $(\sim 160)$, which can restrict its applicability.

The cross-correlation based position reconstruction algorithm [30] is a two-step approach in which a cross-correlation based search method is used to locate the tracer particle position, and a semi-empirical model relating counts to the position of the tracer particle is used for further mesh refinement. This semi-empirical model is a mechanistic simplification of an actual complex mathematical model [30] relating the counts intensity (C) recorded in the detector to the position of the $\gamma$-rays emitting the tracer particle. This model takes into account the effect of the geometry as well as the attenuating medium in between the tracer particle and the detector. Furthermore, it can be implemented on a zone of the bed with its surrounding detectors, which helps minimize the effects of the spread of the counts due to high attenuation medium. It has been found to work satisfactorily in gas- 
solid flows. In this study, RPT calibration experiments suggested that counts received at the detectors are not only a function of distance between the tracer and the detector but also of the attenuation characteristics of a medium in between the tracer and the detector. Hence, a cross-correlation based position reconstruction algorithm was used in this PBR study. The Cross-correlation algorithm consists of the following two steps:

Step I - Finding the cross-correlation coefficient.

The series of counts obtained in all the detectors for some known position of a tracer particle during a calibration $\left(\mathrm{C}_{\text {calib }}\right)$ and similar series of counts obtained during an actual experiment $\left(\mathrm{C}_{\text {run }}\right)$ at a given instant of time can be analyzed to provide an estimate of match between the two counts series. This is quantified in terms of a cross-correlation coefficient $(R)$ (Equation 1). The zero lag of a cross-correlation function is an autocorrelation function, which has maximum value of 1.

$R_{\text {run }, \operatorname{calib}_{k}}(0)=\int_{i=1}^{N_{d}} \frac{c_{\text {calib }(i)}}{\int_{j=1}^{N_{d}} c_{\text {calib }(j)}^{2}} \frac{c_{\text {run }(i)}}{\int_{j=1}^{N_{d}} c_{\text {run }(j)}^{2}}$

Where $C_{\text {calib(i), }}$, series of counts obtained in detector $i=1$ to $N_{d}$ at a given tracer position during a calibration experiment and $\mathrm{C}_{\text {run(i) }}$ series of counts obtained in detector $\mathrm{i}=1$ to $\mathrm{N}_{\mathrm{d}}$, where $\mathbf{N}_{\mathbf{d}}$ is the total number of detectors.

Hence, when the unknown tracer position during an actual experiment is the same as that of a known calibration position, zero lag of normalized cross-correlation function will be equal to one. Therefore, the problem of finding the unknown tracer position is reduced to matching the counts data received in all the detectors to the information obtained for a known calibration position. This is step I of a cross-correlation based position reconstruction algorithm. 
Step II - Establishing additional calibration datasets at a refined level using a semi-empirical model.

Step II is the fitting of the simplified mathematical model over the region of interest (ROI) to refine the experimental calibration grid and establish additional calibration datasets. RPT calibration is time consuming, labor intensive, and is carried out at finite number of positions depending on accessibility to the system. During actual RPT experiments, the tracer particle follows the dynamics of the tracking phase and visits locations in the systems which are usually different than experimental calibration positions. Hence, there is a need to derive additional calibration datasets using RPT calibration experiments and a suitable mathematical model. The newly established calibration datasets at refined mesh level along with in-situ experimental calibration datasets can then solve the problem of identifying the unknown tracer position based on the counts recorded in the detectors. A semi-empirical model (Equation 2), which was proposed and developed by [30] to derive additional calibration data sets. This semi-empirical model is a mechanistic simplification of an actual complex mathematical model relating the counts intensity $(C)$ to the position of the tracer particle emitting $\gamma$-radiation.

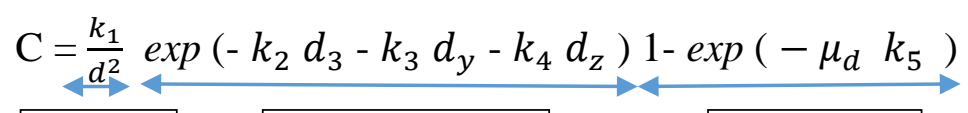

Where, $\mathrm{C}$ - Counts recorded in the detector $\mathbf{k}_{1,2,3,4,5}$ - Model fitted parameters 
$\mathbf{k}_{1}$ - Model fitted parameter proportional to the solid angle subtended by the detector at the tracer location (units $-\mathbf{c m}^{2}$ )

$k_{2,3,4}-$ Effective mass attenuation coefficients (as fitted parameters) of the medium in between the tracer and the detector in $\mathrm{x}, \mathrm{y}$ and $\mathrm{z}$ directions, respectively (units $-1 / \mathrm{cm}$ ) d - Distance of the tracer from the center of the detector crystal (units - cm) $d_{x}, d_{y}, d_{z}-x, y$ and $z$ components of the distance of the tracer from the center of detector crystal (units - cm)

$\mu_{d}$ - Mass attenuation coefficient of the detector material (units $-1 / \mathrm{cm}$ )

$k_{5}$-Length of travel of the photon in the detector crystal (units - cm)

This model takes into account the geometry (through model parameter $k_{1}$ ) as well as the attenuating medium effects in between the tracer and the detector (through model parameters $k_{2,3,4,5}$. Term 1 of Equation 2 is corresponds to the inverse square law and $k_{1}$ is a parameter representing the solid angle subtended by the detector at the tracer location. According to inverse square law, counts intensity is inversely proportional to the square of the tracer-detector distance. Term 2 corresponds to the attenuation characteristics of a heterogeneous medium in between the tracer and the detector. The effective mass attenuation coefficients in $x, y$, and $z$ directions are $k_{2,3,4}$, respectively. Term 3 corresponds to the detector efficiency. The parameter corresponding to the travel length of the photon in the detector material is $\mathbf{k}_{5}$. In this manner, this semi-empirical model takes into account geometry as well as the attenuation characteristics of a medium in between the tracer and the detector and the detector efficiency. Figure 7 illustrates this two-step approach of cross-correlation based position reconstruction algorithm. Step I of the crosscorrelation based position reconstruction algorithm finds the cross correlation coefficient 
((0)) using Equation 1 for each experimental calibration data point and finds the region of interest (ROI) from the whole domain, which helps in minimizing the effects of counts spreading due to the presence of high attenuation materials. It involves finding initial best estimate (IBE) point with the maximum value of cross correlation coefficient $(R(0))$ and then finding neighboring points around it to form the ROI. In Step II, a semi-empirical model which is a mechanistic simplification of the actual complex mathematical model is

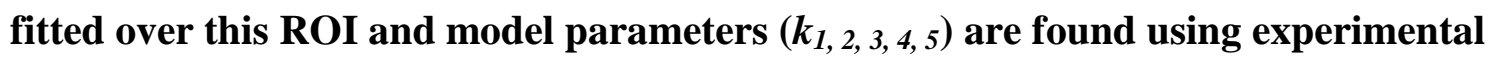
calibration data. These model parameters are then used to establish additional refined calibration data points at finer grid level. Typically, a finer grid of estimated calibration points $\left(\Delta \mathrm{r}=10 \mathrm{~mm}\right.$ (in radial direction), $\Delta \theta=15^{\circ}$ (in azimuthal direction), $\Delta z=5 \mathrm{~mm}$ (in axial direction) is established in step II. After establishing additional calibration datasets, step I is repeated and a point with the maximum value of $R(0)$ is discovered. This two-step process is repeated until convergence criterion of $1-(0) \leq 0.005$ is achieved. This is done by choosing a point with the second maximum value of cross-correlation coefficient as the IBE point, and forming ROI around it and repeating the two-step process. This approach provided satisfactory results and met convergence criterion. This entire two-step approach was implemented in a single MATLAB program and was used to reconstruct the unknown position of a tracer particle. Before applying this cross-correlation based position reconstruction algorithm on an actual RPT experimental dataset, it was necessary to validate this algorithm and estimate reconstruction errors. This was carried out by treating counts data corresponding to some of the known calibration positions as unknown test datasets. This test data was removed from calibration dataset in order to necessitate second step of this position reconstruction algorithm. The obtained validation results of position 
reconstruction algorithm for test datasets are plotted in Figure 8. Results of this validation exercise are also tabulated in Table 2. In addition, the position reconstruction error is shown in Figure 9. The reconstruction errors obtained using this reconstruction algorithm were less than $0.5 \mathrm{~cm}$ (up to $5 \%$ error). This position reconstruction algorithm was then applied on RPT experimental data to study pebbles motion. Figure 10a shows the typical calibration curve for any RPT detector obtained during the study of the granular flow in a PBMR, and Figure 10b shows the estimated calibration datasets after mesh refinement. Estimated calibration datasets in Figure $10 \mathrm{~b}$ also exhibits a 'band effect', which was also seen in the detector calibration curve. After reconstructing the particle position, it is possible to determine the pebble flow field related parameters.

\section{RPT Experiments Trajectories Results}

The lagrangian trajectory of the radioactive tracer can be reconstructed using calibration curves and the cross-correlation based position reconstruction algorithm. The obtained results about tracer trajectories in two and three dimensions, and the velocity vector plot for different initial seeding positions are shown in Figures 11 and 12. A plug-type flow was observed in the upper cylindrical region of the reactor for all seeding positions. The tracer seeded at the center followed a shortest straight line path. Tracers seeded away from the center initially followed a straight line path in the upper portion of the reactor. Afterwards, tracer started moving towards the center of a test reactor and a radial movement of the tracer was observed (Figures 11 and 12). The tracer seeded close to the wall followed the longest path. The length of the tracer trajectory was calculated for each seeding position. A shortest trajectory length of $26.74 \mathrm{~cm}$ was found for the initial seeding position at the center ( $\mathrm{r} / \mathrm{R}$ of 0$)$. The tracer trajectory length increased with change in the dimensionless initial seeding position from the center towards the outer periphery. 
A highest trajectory length of $35.44 \mathrm{~cm}$ (32.54\% increase with respect to shortest trajectory length) was observed for the initial tracer seeding position close to the wall ( $\mathrm{r} / \mathrm{R}$ of 0.92$)$. The values of trajectory length and percentage increase with respect to the shortest trajectory length are tabulated in Table 3.

Figure 13 shows the position of the tracer (radioactive particle) for different initial seeding positions as a function of time. A comparison of tracer $\mathrm{z}$ coordinates for same amount of travel time suggested the observance of plug flow type movement (at the end of 5 hours). The difference in tracer $\mathrm{z}$ coordinates come much more pronounced at the end of eight hours. Also, some radial movement of tracer is observed for all the seed positions except when seeded at the center. Hence, it is clear from time-dependent tracer position information that the tracer seeded at the center moved much faster than when seeded close to the wall. This also suggests that the overall residence time should be lowest for the tracer seeded at the center, whereas it should be highest when tracer is seeded close to the wall.

In addition, RPT results were analyzed to estimate the tracer velocities. Figure 14a shows locations of control volumes (CV) 1 and 2. These control volumes are $1 \mathrm{~cm}$ thick and are located at a depth of $11 \mathrm{~cm}(\mathrm{CV} 1)$ and $27 \mathrm{~cm}(\mathrm{CV} 2)$ from the top surface of the test reactor. Obtained velocity results for CV1 and CV2 are shown in Figure 14b. A plug-flow type velocity radial profile was observed for $\mathrm{CV} 1$ in the upper region. The velocity profile is nearly uniform except in a region close to the wall. This suggests that pebbles move collectively as a solid mass in the upper region. A velocity radial profile with pronounced concavity near the central region was observed for CV 2. This indicates that the tracer that was initially seeded at the center ( $\mathrm{r} / \mathrm{R}$ of 0$)$ moves much faster than when it was initially seeded near the wall ( $\mathrm{r} / \mathrm{R}$ of 0.92$)$. 
This observation is consistent with the pebble bed modular reactor( PBMR) safety analysis report [34]. Many previous studies failed to capture this pronounced concavity in the velocity radial profile. The main possible reason for not capturing this effect could be the use of a $180^{\circ}$ half model, which introduces 'wall-effect'. Also, values of mass flow index (MFI), which is calculated using Equation 3 [35] were found for both CVs.

$\mathrm{MFI}=\frac{\text { Velocity close to the wall }\left(V_{\text {wall }}\right)}{\text { Velocity at the center }\left(V_{\text {center }}\right)}$

The mass flow was observed for values of the mass flow index (MFI) greater than 0.3, and the funnel flow was observed for values of mass flow index less than 0.3 [36]. In mass flow, there is a simultaneous motion of all the particles as a solid mass. In funnel/core flow, there is a rapid movement of particles, which are surrounded either by slowly moving or stagnant particles in the center. The obtained value of MFI for CV1 suggests that there is a mass flow suggesting a simultaneous motion of all particles as a solid mass. Also, the obtained value of MFI for CV2 suggests that there is a funnel flow indicating that the particles in the center are moving much faster than the outer ones close to the wall. The obtained experimental results from RPT technique serve as benchmark data for assessing the contact force models used in the discrete element method (DEM) based simulations.

\section{Remarks}

The following are the highlights of the work carried out and the key findings of this study:

- The RPT experiments were carried out around the continuous pebbles recirculation experimental set-up that mimics a cold flow moving bed operation of the Pebble Bed Modular Reactor. 
- A co-60 based radioactive tracer particle mimicking glass marbles in terms of shape, diameter and density was used for RPT experiments.

- The RPT calibration data that was collected suggested that detector counts are not only a function of the tracer-detector distance, but also of the attenuation characteristics of the medium in between the source and the detector.

- A cross-correlation based position reconstruction algorithm along with a simplified mechanistic model, which takes into account the geometry as well as the attenuating medium effects was used for the RPT experiments position reconstruction step.

- Velocity radial profile results obtained using the RPT technique suggested a plug flow type velocity profile in the upper cylindrical region, whereas velocity profile with pronounced concavity was observed near the cylinder-cone transition point, which is consistent with predictions based on mass flow index calculations and previous studies.

- RPT experiment results provided benchmark data, which can be used for validation of DEM based simulation results 
NOMECLATURE

\author{
Symbol \\ Description \\ $\mathrm{C}$ \\ Counts recorded in the detector \\ $\mathrm{N}_{\mathrm{d}}$ \\ The number of detectors \\ $R_{\text {run, calib }}$ \\ Cross-correlation coefficient \\ $\mathrm{k}_{1,2,3,4,5}$ \\ Model fitted parameters \\ $k 1$ \\ Model fitted parameter proportional to the solid angle subtended by \\ The detector at the tracer location (units $-\mathrm{cm}^{2}$ ) \\ $k 2,3,4$ \\ Effective mass attenuation coefficients (as fitted parameters) of the \\ medium in between the tracer and the detector in $\mathrm{x}, \mathrm{y}$ and $\mathrm{z}$ \\ directions respectively (units $-1 / \mathrm{cm}$ ). \\ d \\ Distance of the tracer from the center of the detector crystal (units \\ $\mathrm{cm})$. \\ $d_{x}, d_{y}, d_{z}$ \\ $\mathrm{x}, \mathrm{y}$ and $\mathrm{z}$ components of the distance of the tracer from the center of \\ detector crystal (units $-\mathrm{cm}$ ). \\ $k 5$ \\ Length of travel of the photon in the detector crystal (units $-\mathrm{cm}$ ) \\ $\mathrm{V}_{\text {wall }}$ \\ Velocity closes to wall \\ $\mathrm{V}_{\text {Center }}$ \\ Velocity closes to center \\ $\mu_{d}$ \\ Mass attenuation coefficient of the detector material (units $-1 / \mathrm{cm}$ )
}

Dimensionless groups

$\mathrm{r} / \mathrm{R}$

Dimensionless radial position

\title{
Abbreviations
}

PBMR

Pebble Bed Modular Reactor

VHTR

Very High Temperature Reactor

RPT

Radioactive Particle Tracking

DEM

Discrete Element Method

PBR

Prismatic Block Reactor 


\section{Acknowledgements}

The authors acknowledge the financial support provided by Department of Energy (DOE)

Nuclear Energy Research Initiative (NERI) project (NERI-08-043).

\section{References}

1. Boer, B., Optimized core design and fuel management of a pebble-bed type nuclear reactor. 2009: Delft University of Technology,Delft, Netherlands

2. F.C.Gatt, Flow of spheres and near spheres in cylindrical vessels, part IV, in Australian Energy commission. 1973: Lucas Heights

3. G.P.Deutsch, Flow of granular material from silos, in movement of Gran. Material and Struct., Fluid flow and heat transfer in packed beds. 1967: AAECRE Lucas heights

4. A.Shehata, A New Method for Radioactive Particle Tracking. 2005, North Carolina State University, Raleigh, North Carolina.

5. M.Z.Bazant, A.C.K.a., Pebble flow experiments for pebble bed reactors, in 2nd International Topical meeting on High Temperature Reactor Technology 2004: Beijing,China.

6. Y.Li, Y.X., S.Jiang, DEM simulations and experiments of pebble flow with monosized spheres, Powder Technology 2009. 193(3): p. 312-318

7. Y.L.Sun, Y.H.X.a., Status of the HTR programme in China, in IAEA TCM on High Temperature Gas Cooled Reactor Application and Future Prospects ECN. 1997: Petten,The Netherlands.

8. S.Y. Jiang, X.T.Y., Z.W.Tang,W.J.Wang,J.Y.Tu,Z.Y.Liu,J.Li, Experimental and numerical validation of a two-region-designed pebble bed reactor with dynamic core. Nuclear Engineering and Design, 2012. 246: p. 277-285.

9. Z.Wang, A Dual Measurement System for Radioactive Tracer Pebble Tracking in PBRs. 2011, North Carolina State University, Raleigh, North Carolina

10. C.Rycroft, G.G., J.Landry., and M.Bazant, Analysis of granular flow in a pebblebed nuclear reactor. Phys. Rev. E., 2006. 74.

11. KHANE, V.B., EXPERIMENTAL AND COMPUTATIONAL INVESTIGATION OF FLOW OF PEBBLES IN A PEBBLE BED NUCLEAR REACTOR. 2014, MISSOURI UNIVERSITY OF SCIENCE AND TECHNOLOGY.

12. N.Rados, A.S., and M. H.Al-Dahhan, Solids Flow Mapping in High Pressure Slurry Bubble Columns using CARPT. Chem. Eng. Sci., 2005. 60(22): p. 6067-6072.

13. M.Vesavikar, Understanding hydrodynamics and performance of anaerobic digesters. 2006, Washington University, St. Louis ,Missouri. 
14. R.Varma, M.A.-D., Effect of sparger design on hydrodynamics of a gas recirculation anaerobic bioreactor. Biotechnology and Bioengineering, 2007. 98(6): p. 1146-1160.

15. J.Chaouki, F.C., M.P.Duduković, Noninvasive Tomographic and Velocimetric Monitoring of Multiphase Flows. Ind. Eng. Chem. Res., 1997. 36: p. 4476-4503.

16. S. Roy, A.K., M.H. Al-Dahhan, M.P. Dudukovic, Experimental investigation of the hydrodynamics in a liquid-solid riser. AIChE J., 2005. 51(3): p. 802-835.

17. S. Bhusarapu, M.H.A.-D.a.M.P.D., Solids Flow Mapping in a Gas-Solid Riser: Velocity and Holdup Profiles. Powder Technology, 2006. 163: p. 98-123.

18. Al-Dahhan, A.S.a.M.H., A New Methodology for Hydrodynamic Similarity in Bubble Column Reactors. Can. J. Chem. Eng, 2010. 88: p. 503-517.

19. N.Devnathan, Investigation of Liquid Hydrodynamics in Bubble columns via Computer Automated Radioactive Particle Tracking (CARPT). 1991, Washington University St. Louis Missouri

20. S.Degaleesan, Fluid dynamic measurements and modeling of liquid mixing in bubble columns. 1997, Washington University, St.Louis, Missouri

21. Rammohan, A., Characterization of single and multiphase flows in stirred tank reactors. 2002, Washington University, Saint Louis, Missouri

22. C.Ong, B., Experimental investigation of bubble columns hydrodynamics: effect of elevated pressure and superficial gas velocity. 2003, Washington University, Saint Louis

23. S.Bhusarapu, Solids flow mapping in gas solid risers. 2005, Washington University St. Louis Missouri.

24. Ashfaq Shaikh, M.H.A.-D., A review on flow regime transition in bubble columns. International Journal of Chemical Reactor Engineering, 2007. 5.

25. Han, Hydrodynamics, back-mixing, and Mass Transfer in a Slurry Bubble Column Reactor for Fischer-Tropsch Alternative Fuels. 2007, Washington University, Saint Louis, Missouri (2007).

26. S.Roy, F.L., M.H.Al-Dahhan, M.P.Duduković, Optimal design of radioactive particle tracking experiments for flow mapping in opaque multiphase reactors. Applied Radiation and Isotopes, 2002. 56: p. 485-503

27. Godfroy, L., Larachi, F., Kennedy, G., Grandjean, B., Chaouki, J.,, On-line flow visualization in multiphase reactors using neural networks. Applied Radiation and Isotopes, 1997. 48(2): p. 225235.

28. Gupta, P., Churn-turbulent bubble columns: experiments and modeling. 2002, Washington University, St. Louis, Missouri.

29. N.Rados, a.M.H.A.-D., Solids Flow Mapping in High Pressure Slurry Bubble Columns using CARPT. Chem Eng Sci., 2005. 60(22): p. 6067-6072.

30. S.Bhusarapu, Solids flow mapping in gas solid risers. 2005, Washington University St. Louis Missouri.

31. Yang, Y.B.D., N., and Duduković, M.P., , Liquid Backmixing in Bubble Columns via ComputerAutomated Radioactive Particle Tracking (CARPT). Experiments in Fluids, 1993. 16: p. 1-9.

32. Larachi, F., Kennedy, G., Chaouki, J., y-ray detection system for 3-D particle tracking in multiphase reactors. Nuclear Inst. and Methods in Physics Research, 1994: p. 568-576.

33. Godfroy, L., Larachi, F., Chaouki, J.,, Position and velocity of a large particle in a gas/solid riser using the radioactive particle tracking technique. Canadian Journal of Chemical Engineering, 1999. 77(2): p. 253-261.

34. PBMR Safety Analysis Report. 2000.

35. M.Nedderman, R., Statics and Kinematics of Granular Materials. 1992: Cambridge University Press. 
36. J.R.Johanson, A.W.J., Bulletin 116: Stress and velocity fields in gravity flow of bulk solids 1962: Salt Lake City, UT

\section{List of Tables:}

Table 1 Position coordinate of the RPT detectors.

Table 2 Position reconstruction algorithm validation results.

Table 3 Tracer trajectory length values for different initial seeding positions.

\section{List of Figures:}

Figure 1 Continuous pebble recirculation experimental set-up.

Figure 2 Continuous pebbles recirculation experimental set-up at Missouri S\&T along with implementation of RPT technique.

Figure 3 RPT tracer particle.

Figure 4 RPT implementation around continuous Pebble recirculation experimental set-up.

Figure 5 Manual Calibration Apparatus.

Figure 6 RPT detector calibration curve for PBMR study.

Figure 7 Schematics of two-step position reconstruction approach.

Figure 8 Validation of position reconstruction algorithm results.

Figure 9 Error bar of calibration points.

Figure 10 Estimated calibration datasets after mesh refinement using semi-empirica model. 
Figure 11 RPT results.

Figure 12 Three-dimensional tracer trajectories obtained using RPT.

Figure 13 Tracer positions for different initial seeding positions as a function of time.

Figure 14 Pebbles velocity radial profile obtained using RPT. 
Table 1 Position coordinate of the RPT detectors.

\begin{tabular}{|l|l|l|l|}
\hline Detector & Z, cm & R, cm & $\theta{ }^{{ }^{0}}$ \\
\hline 1 & 7.62 & 22.86 & 112.5 \\
\hline 2 & 15.24 & 22.86 & 67.5 \\
\hline 3 & 22.86 & 22.86 & 112.5 \\
\hline 4 & 30.48 & 22.86 & 67.5 \\
\hline 5 & 7.62 & 22.86 & 22.5 \\
\hline 6 & 15.24 & 22.86 & 337.5 \\
\hline 7 & 22.86 & 22.86 & 22.5 \\
\hline 8 & 30.48 & 22.86 & 337.5 \\
\hline 9 & 7.62 & 22.86 & 202.5 \\
\hline 10 & 15.24 & 22.86 & 157.5 \\
\hline 11 & 22.86 & 22.86 & 202.5 \\
\hline 12 & 30.48 & 22.86 & 157.5 \\
\hline 13 & 7.62 & 22.86 & 292.5 \\
\hline 14 & 15.24 & 22.86 & 247.5 \\
\hline 15 & 22.86 & 22.86 & 292.5 \\
\hline 16 & 30.48 & 22.86 & 247.5 \\
\hline
\end{tabular}

Table 2 Position reconstruction algorithm validation results

\begin{tabular}{|c|c|c|c|c|c|c|c|c|}
\hline \multirow{2}{*}{$\begin{array}{l}\text { Position } \\
\text { number }\end{array}$} & \multicolumn{3}{|c|}{ Actual Position co-ordinate } & \multicolumn{3}{|c|}{$\begin{array}{l}\text { Reconstruction position co- } \\
\text { ordinate }\end{array}$} & \multirow[t]{2}{*}{$\begin{array}{c}\text { Error } \\
\text { ( in cm) }\end{array}$} & \multirow[t]{2}{*}{$\%$ Errol } \\
\hline & $\begin{array}{l}X \\
z\end{array}$ & $\begin{array}{c}\mathrm{y} \\
\text { (in cm) }\end{array}$ & & $\begin{array}{l}X \\
z\end{array}$ & $\begin{array}{r}\mathrm{y} \\
\text { ( in c }\end{array}$ & & & \\
\hline 1 & 13.75 & -2.46 & 9.00 & 13.75 & -2.46 & 9.5 & 0.50 & 5.26 \\
\hline 2 & -13.92 & 1.23 & 17.00 & -13.92 & 1.23 & 17.00 & 0.00 & 0.00 \\
\hline 3 & -8.36 & -11.19 & 27.00 & -8.36 & -11.19 & 27.50 & 0.50 & 1.82 \\
\hline 4 & -2.23 & 4.54 & 11.00 & -2.28 & 4.54 & 11.50 & 0.50 & 4.35 \\
\hline 5 & 3.39 & 3.79 & 17.00 & 3.39 & 3.79 & 17.50 & 0.50 & 2.86 \\
\hline 6 & 5.08 & 0.00 & 27.00 & 5.08 & 0.00 & 26.50 & 0.50 & 1.89 \\
\hline 7 & 0.00 & 0.00 & 9.00 & 0.00 & 0.00 & 9.50 & 0.50 & 5.26 \\
\hline 8 & 0.00 & 0.00 & 17.00 & 0.00 & 0.00 & 17.50 & 0.50 & 2.86 \\
\hline 9 & 0.00 & 0.00 & 27.00 & 0.00 & 0.00 & 26.50 & 0.50 & 1.89 \\
\hline 10 & -0.45 & -10.15 & 15.00 & -0.45 & -10.15 & 27.50 & 0.50 & 1.82 \\
\hline 11 & 1.34 & 10.07 & 15.00 & 1.34 & 10.07 & 14.50 & 0.50 & 3.45 \\
\hline 12 & -7.86 & 6.43 & 9.00 & -7.86 & 6.43 & 9 & 0.50 & 0.00 \\
\hline
\end{tabular}


Table 3 Tracer trajectory length values for different initial seeding positions

\begin{tabular}{|c|c|c|c|c|}
\hline & \multicolumn{4}{|c|}{$\begin{array}{c}\text { Tracer Inlet Seed Position } \\
\text { Dimensionless radial position(r/R) }\end{array}$} \\
\cline { 2 - 5 } & 0 & 0.33 & 0.67 & 0.92 \\
\hline $\begin{array}{c}\text { Trajectory Length } \\
\text { (in cm) }\end{array}$ & 26.74 & 29.23 & 32.4 & 35.44 \\
\hline $\begin{array}{c}\% \text { increase with } \\
\text { respect to shortest } \\
\text { trajectory length }\end{array}$ & -- & 9.31 & 21.17 & 32.54 \\
\hline
\end{tabular}




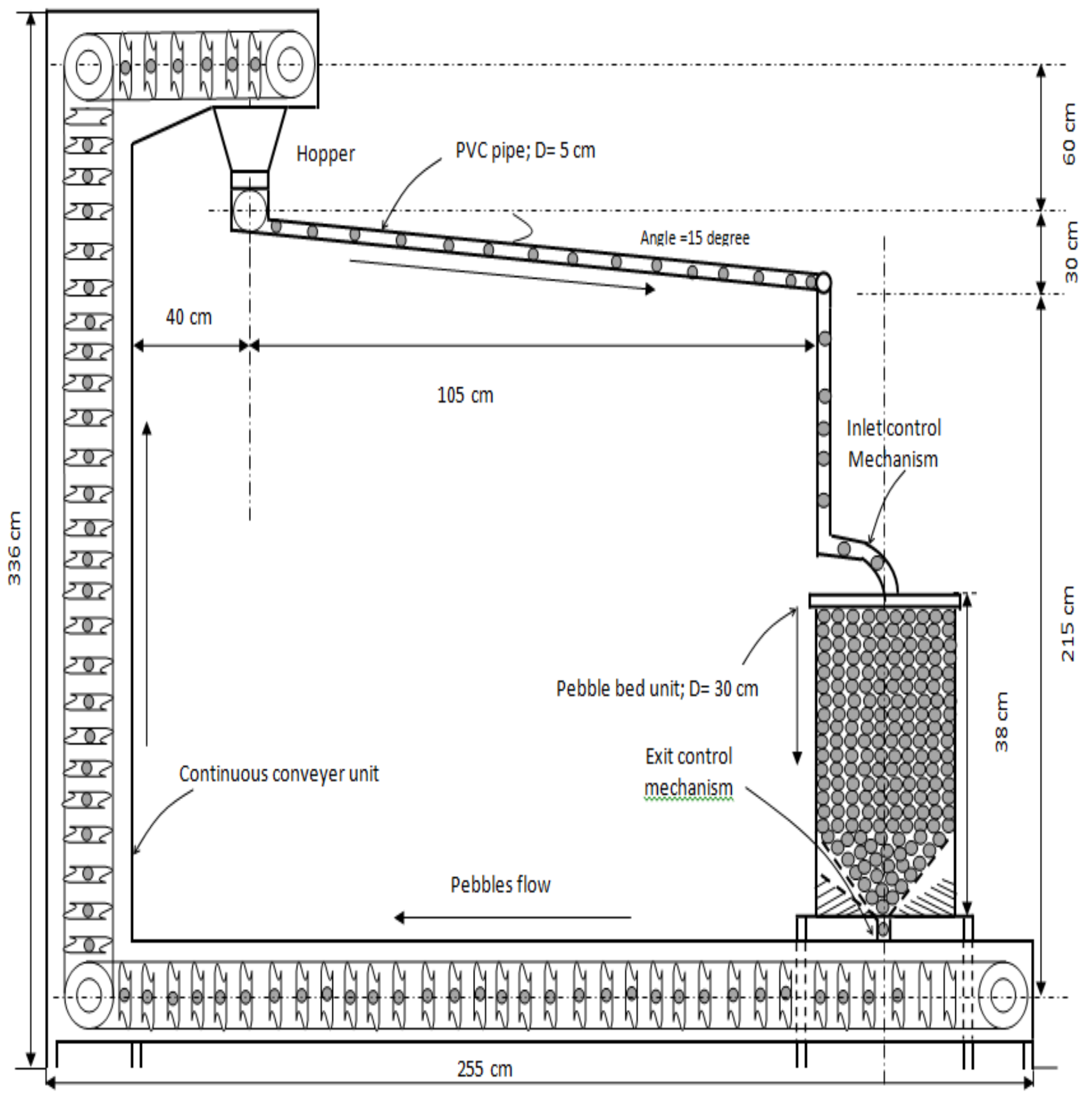

a. Schematic diagram 


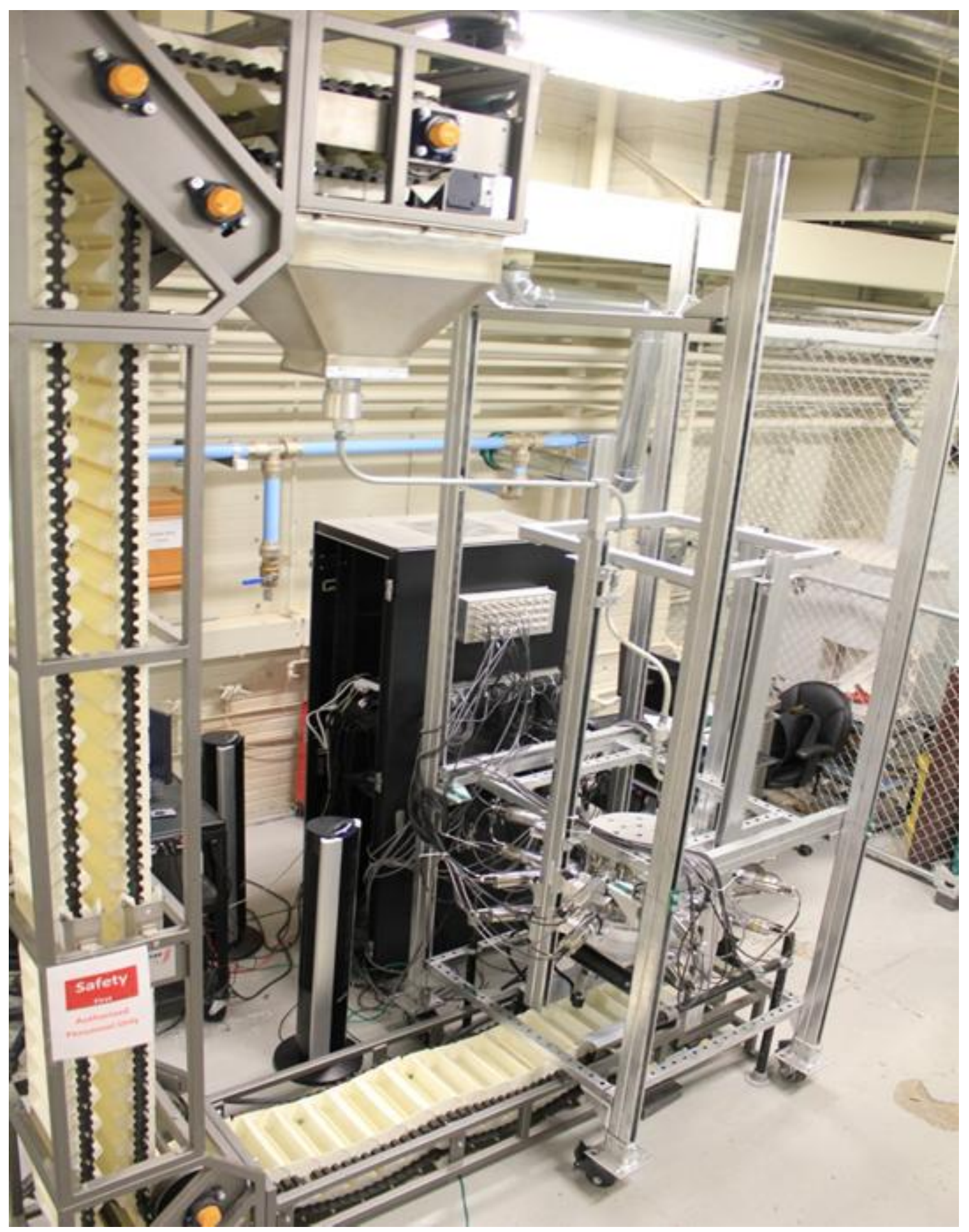

b. Physical picture

Figure 1 Continuous pebble recirculation experimental set-up 


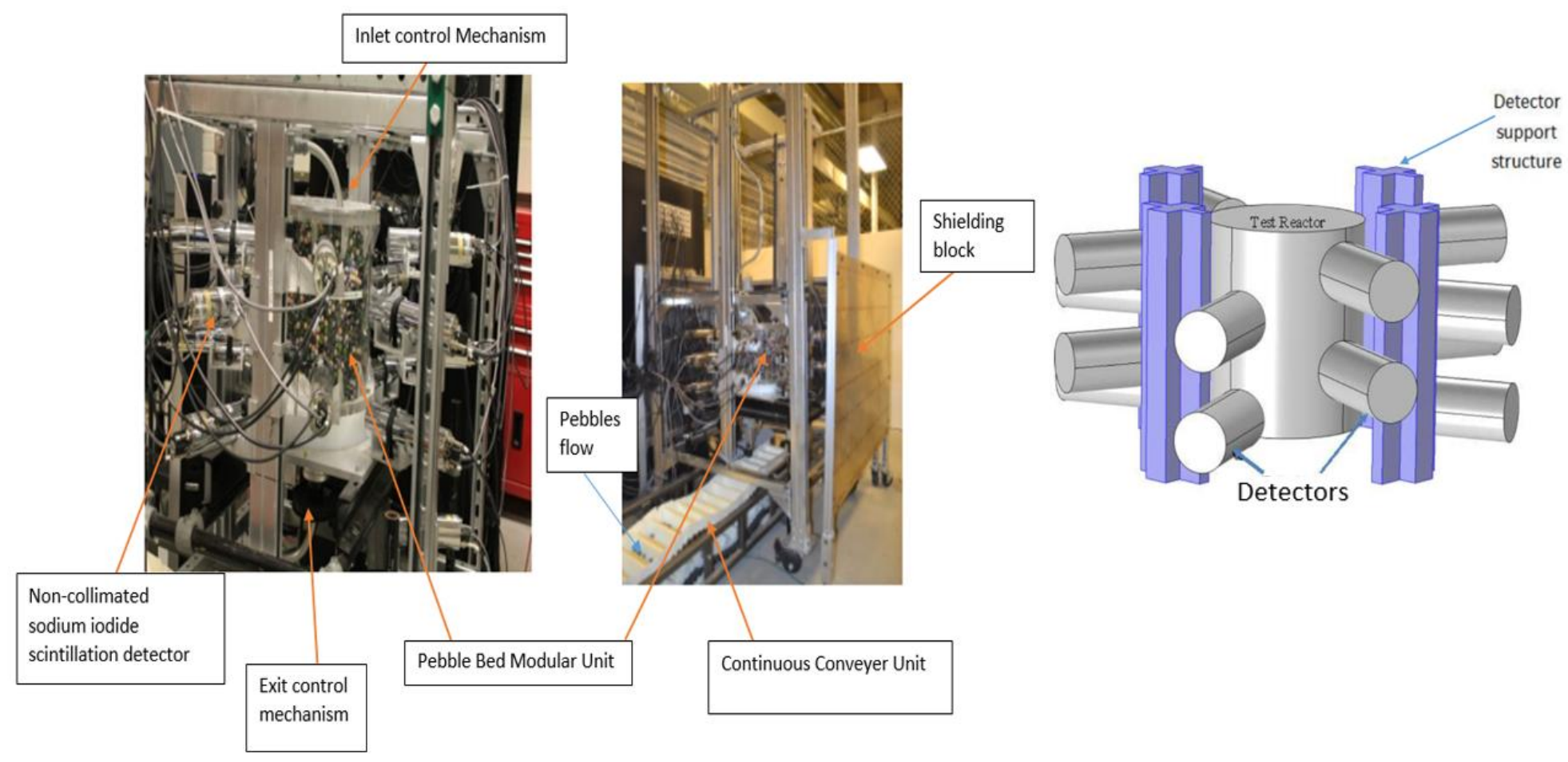

a. Physical Picture

b. Schematic diagram

Figure 2 Continuous pebbles recirculation experimental set-up at Missouri S\&T along with implementation of RPT technique

Tracer

Particle

Air gap of

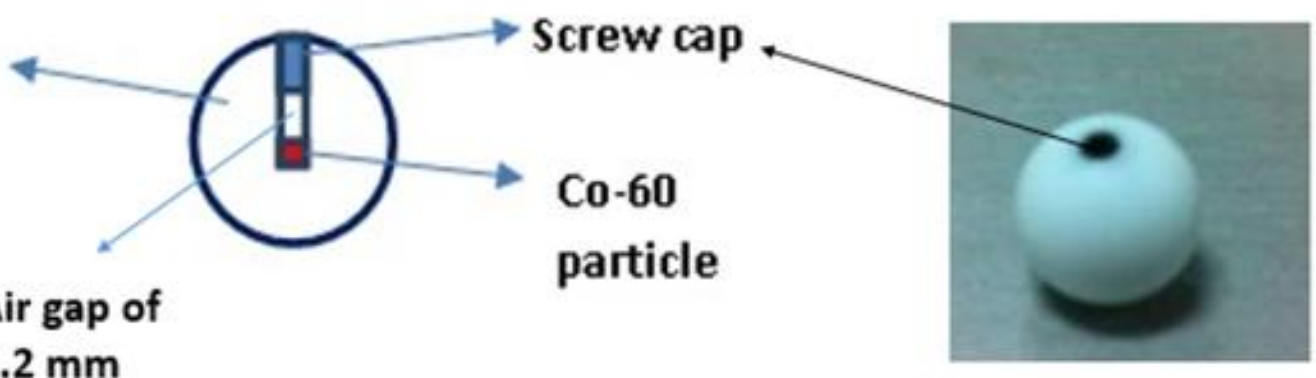

Length

a. Schematic diagram

b. Actual Picture

Figure 3 RPT tracer particle 

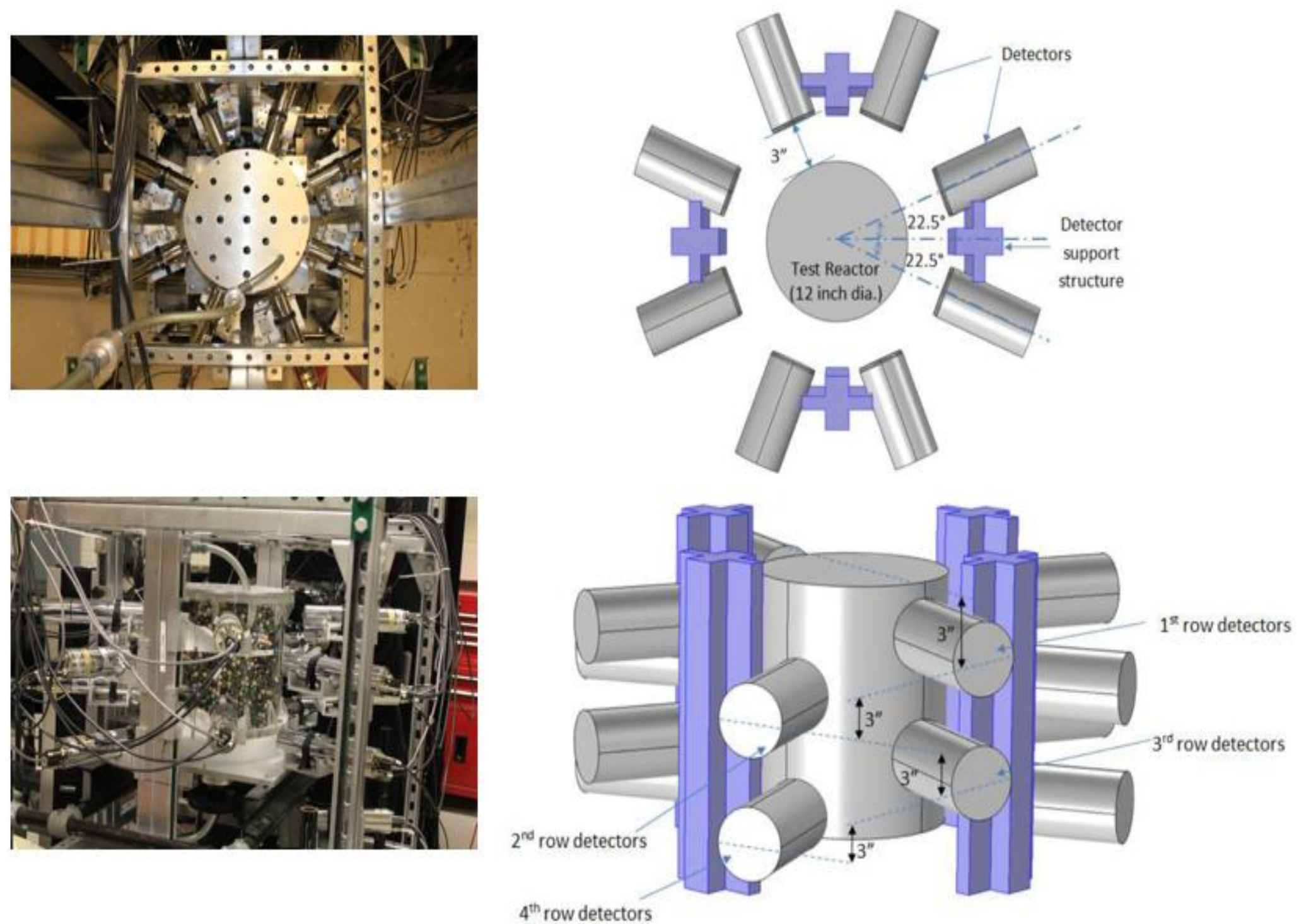

Figure 4 RPT implementation and the detectors arrangement around continuous Pebble recirculation experimental set-up 


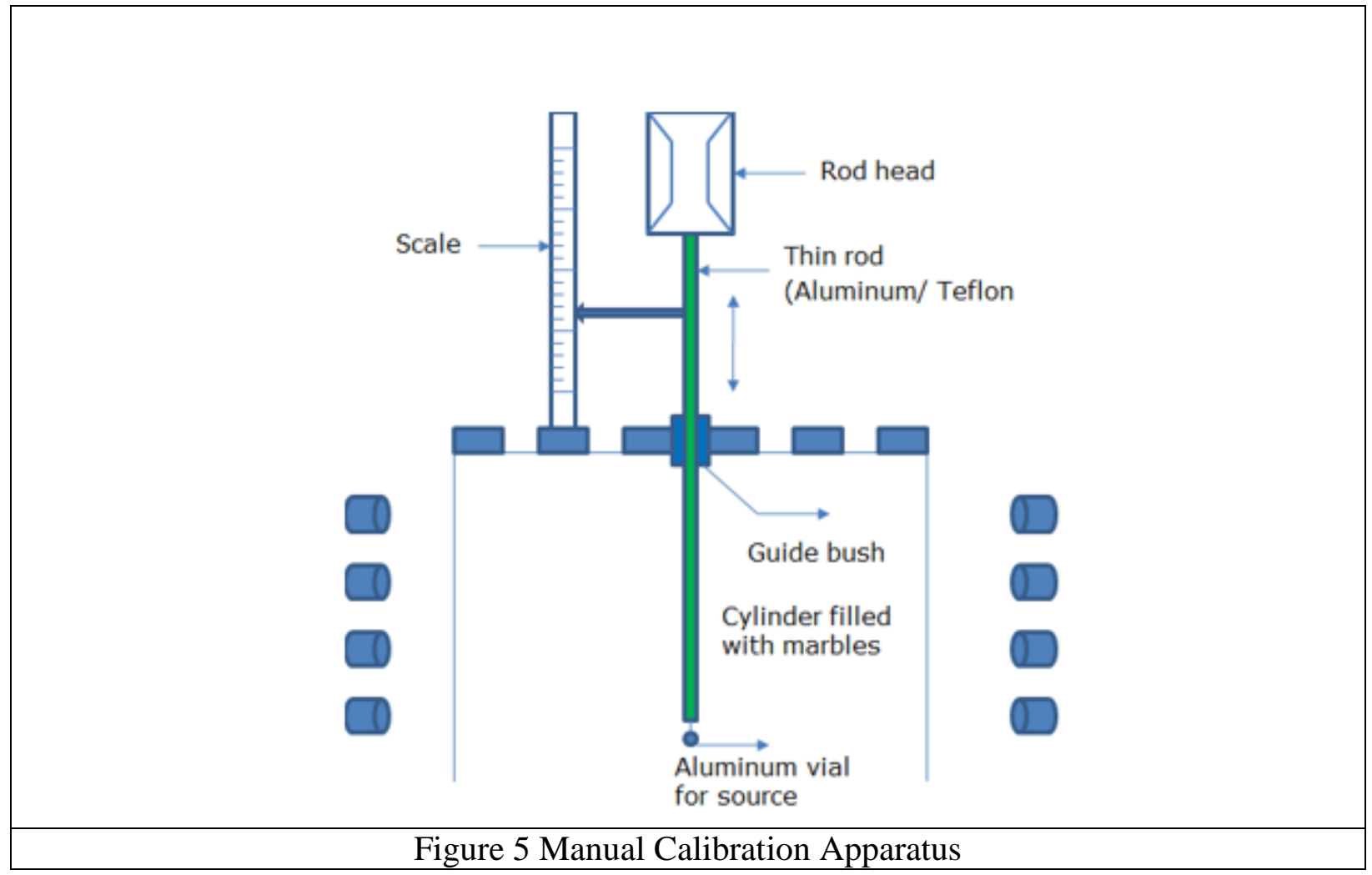




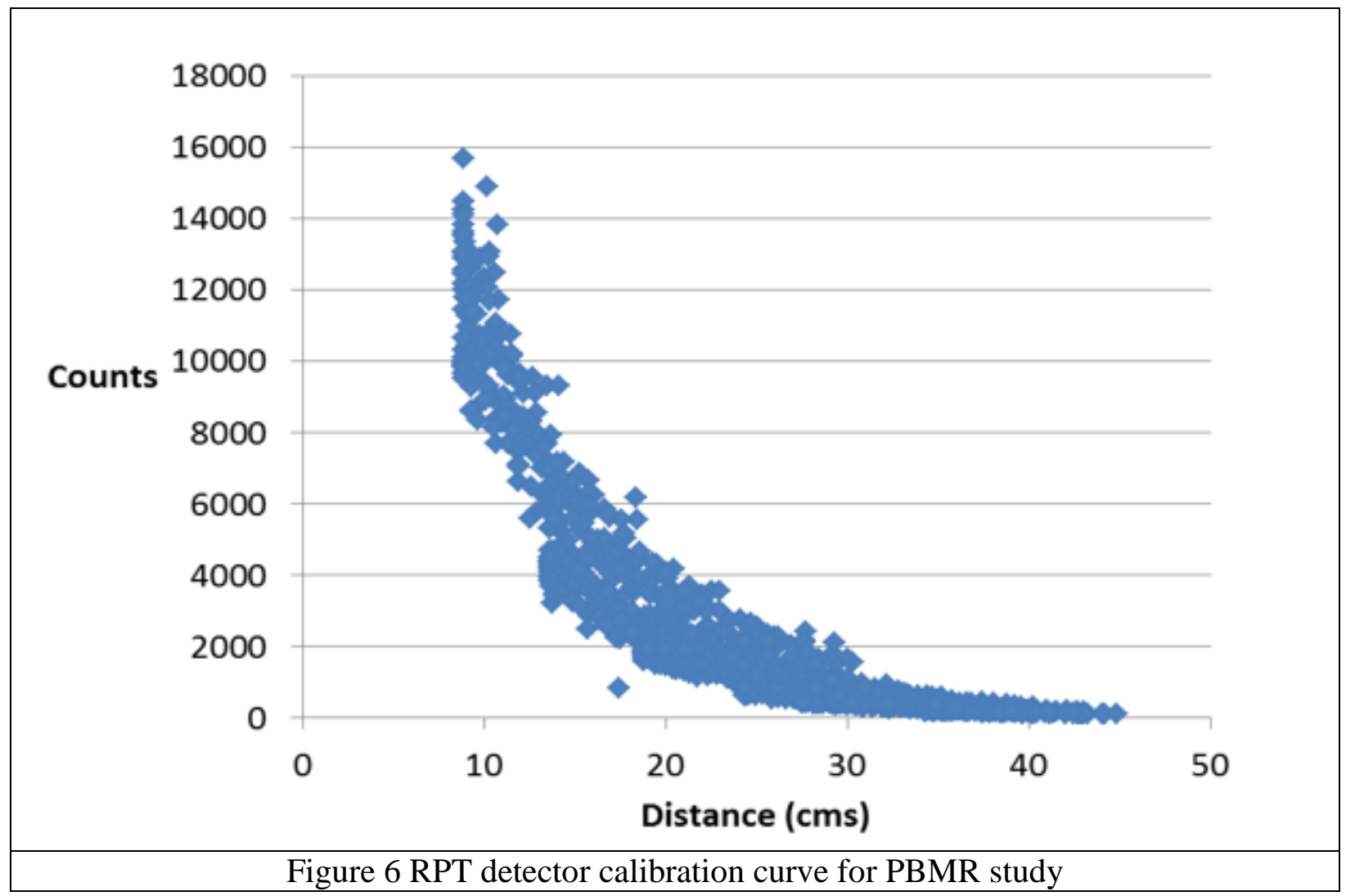




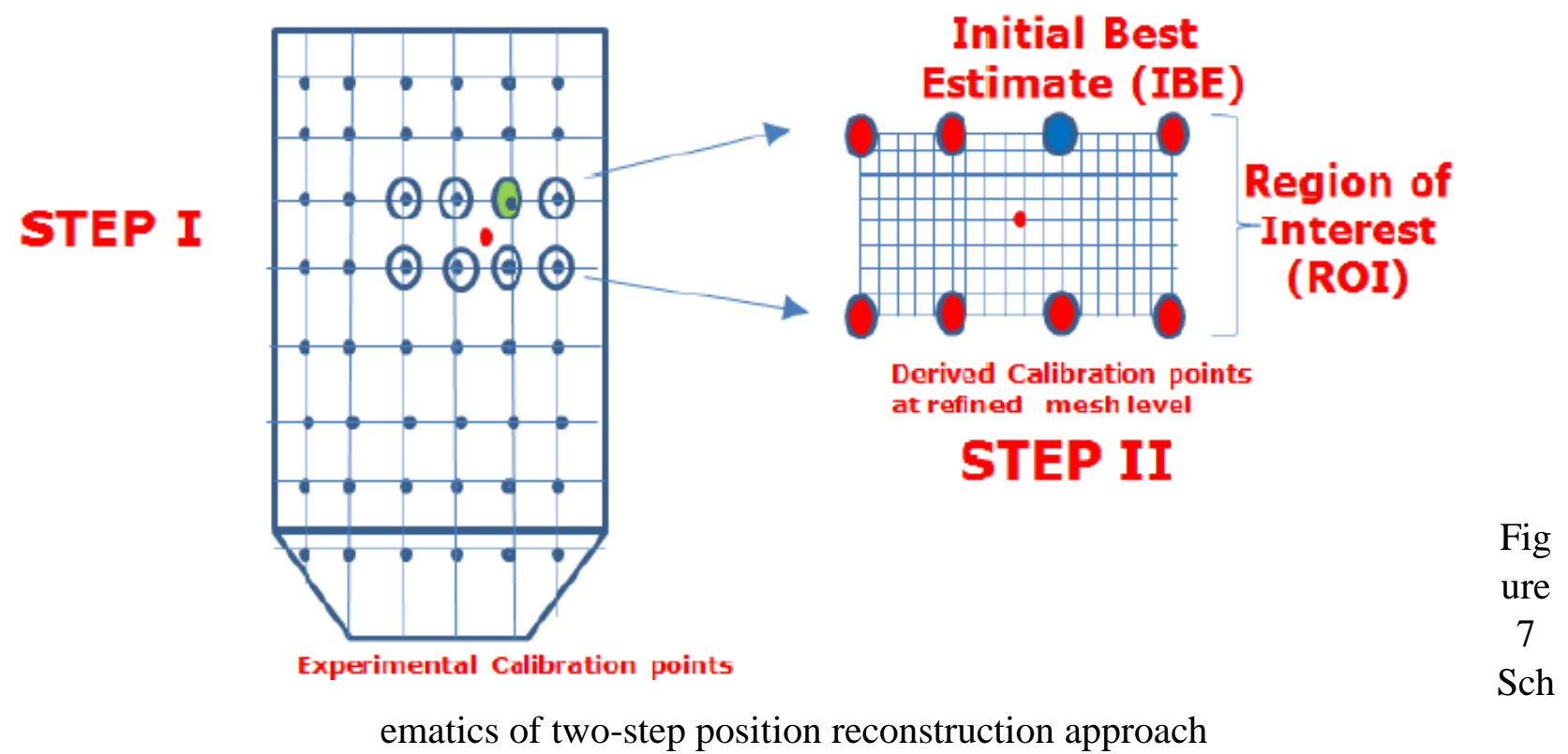




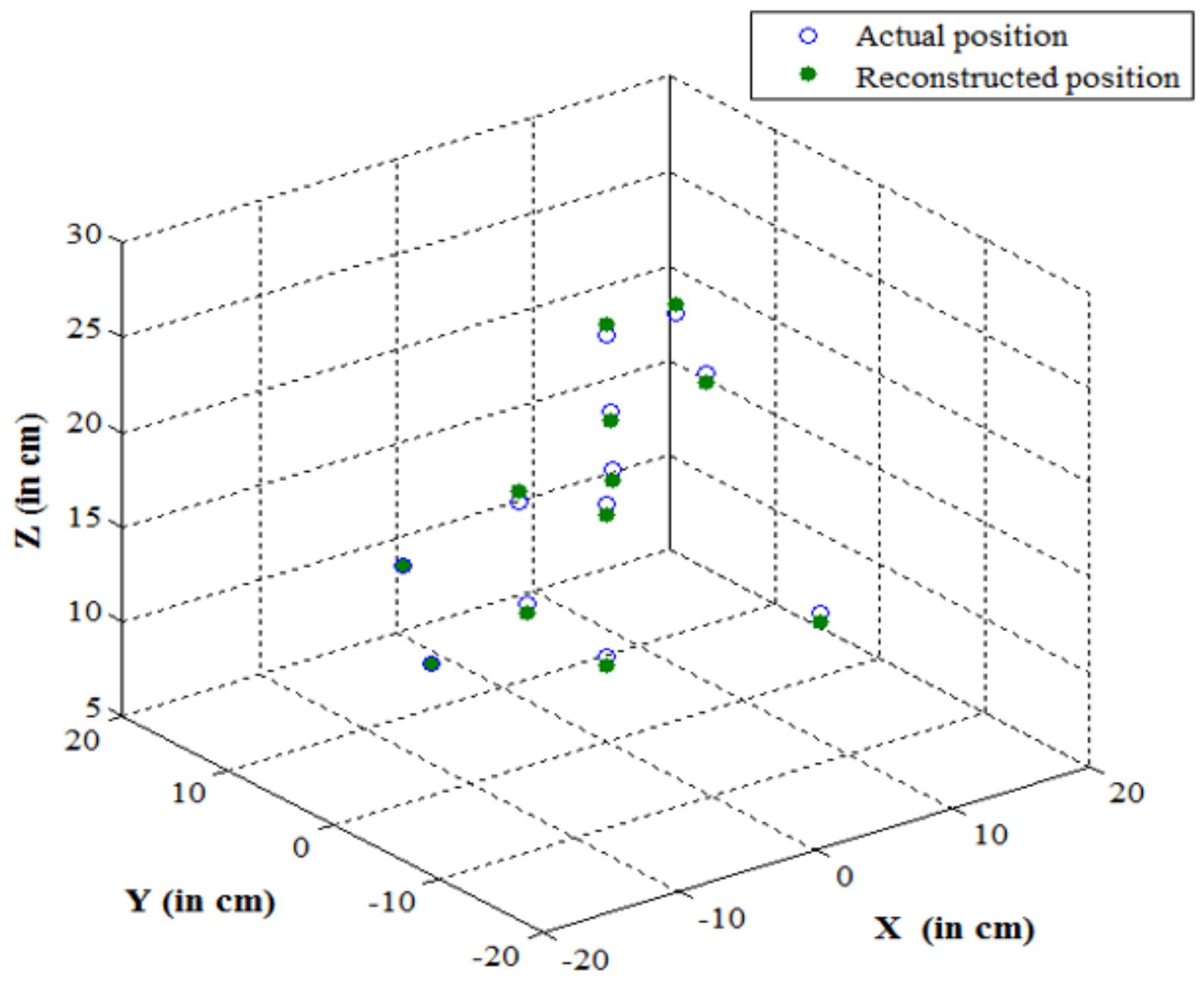

Figure 8 Validation of position reconstruction algorithm results 


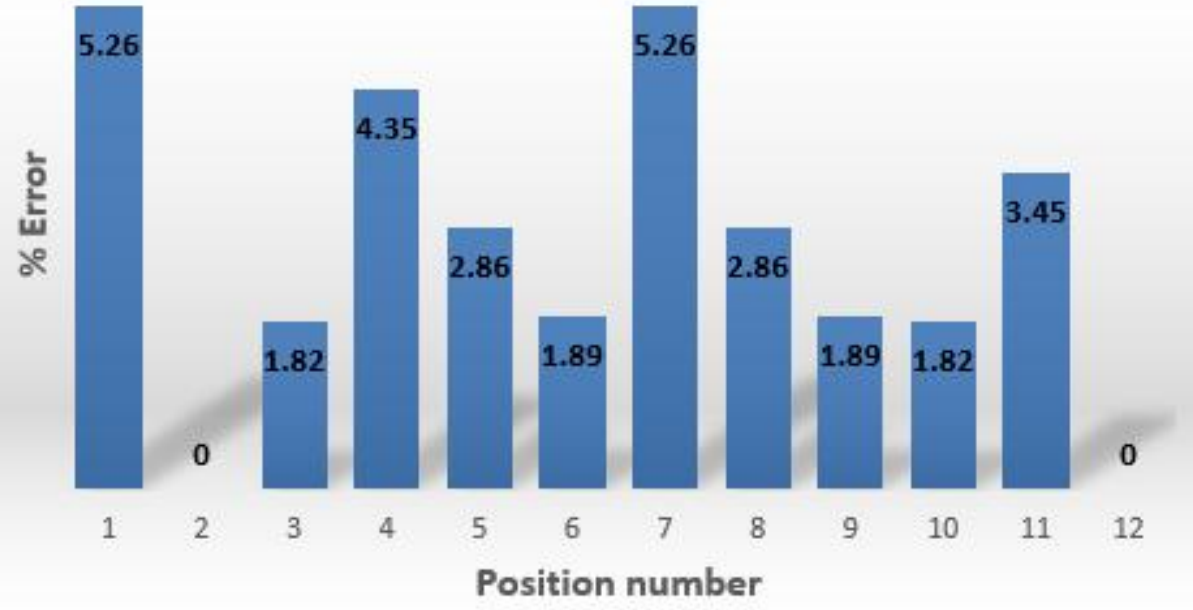

Figure 9 Error bar of calibration points 


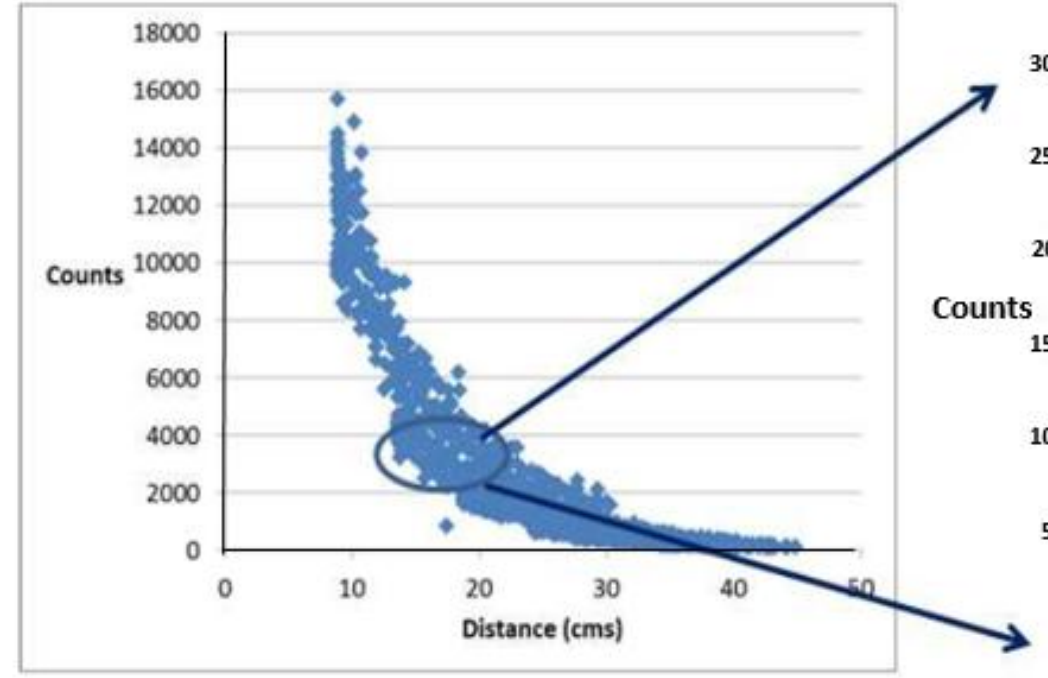

a. Calibration curve

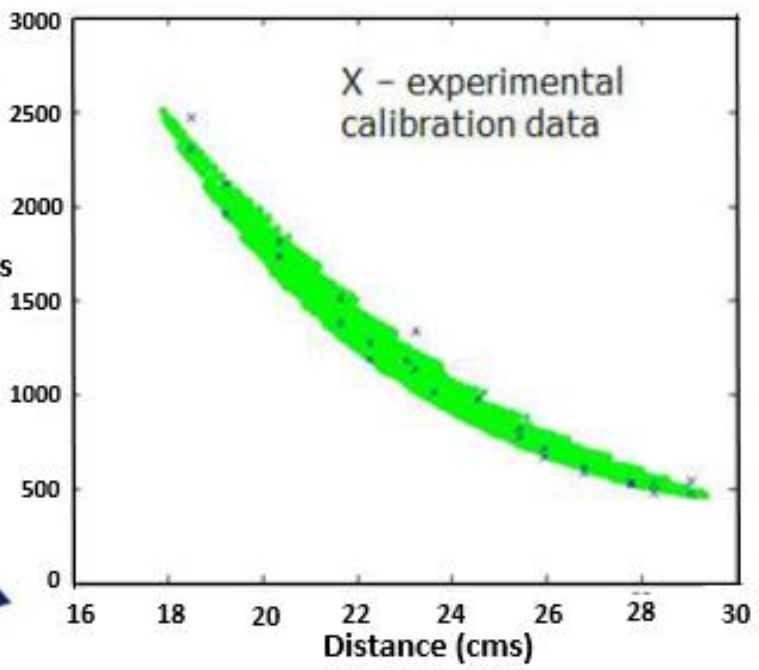

b. Estimated calibration data

Figure 10 Estimated calibration datasets after mesh refinement using semi-empirica model. 


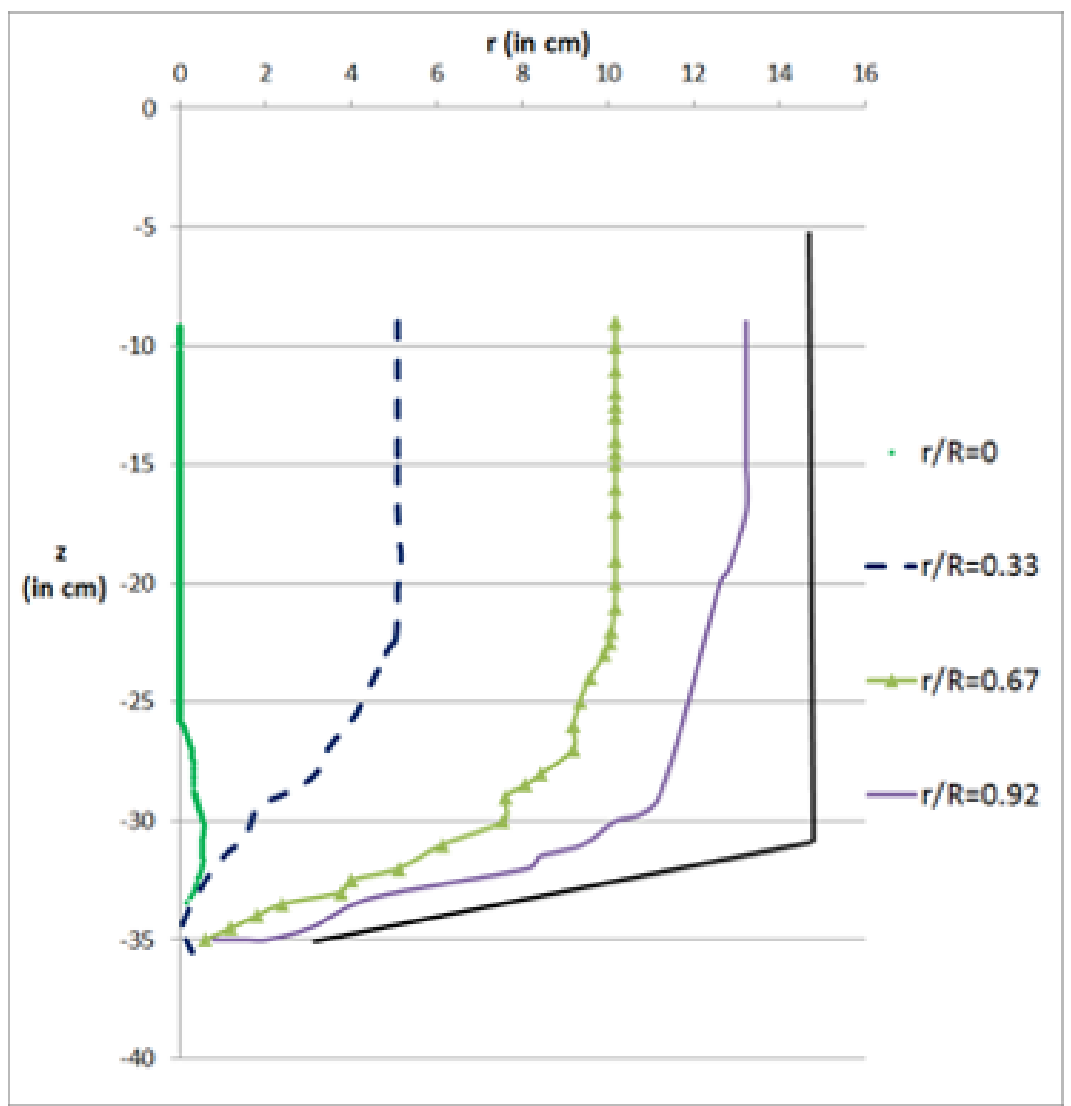

a. Two-dimensional tracer Trajectories obtained using RPT

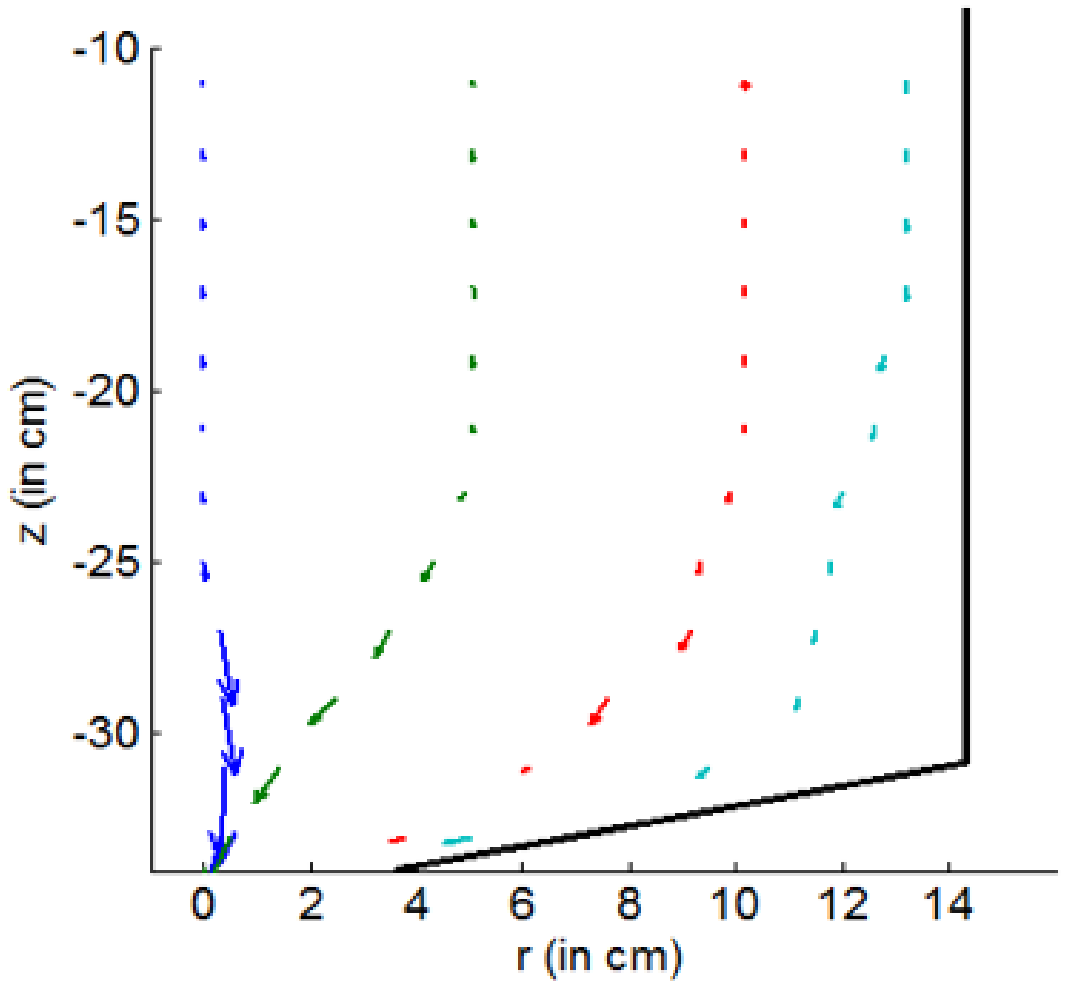

b. Velocity vector plot (Length of arrows proportional to magnitude of velocity)

Figure 11 RPT results 


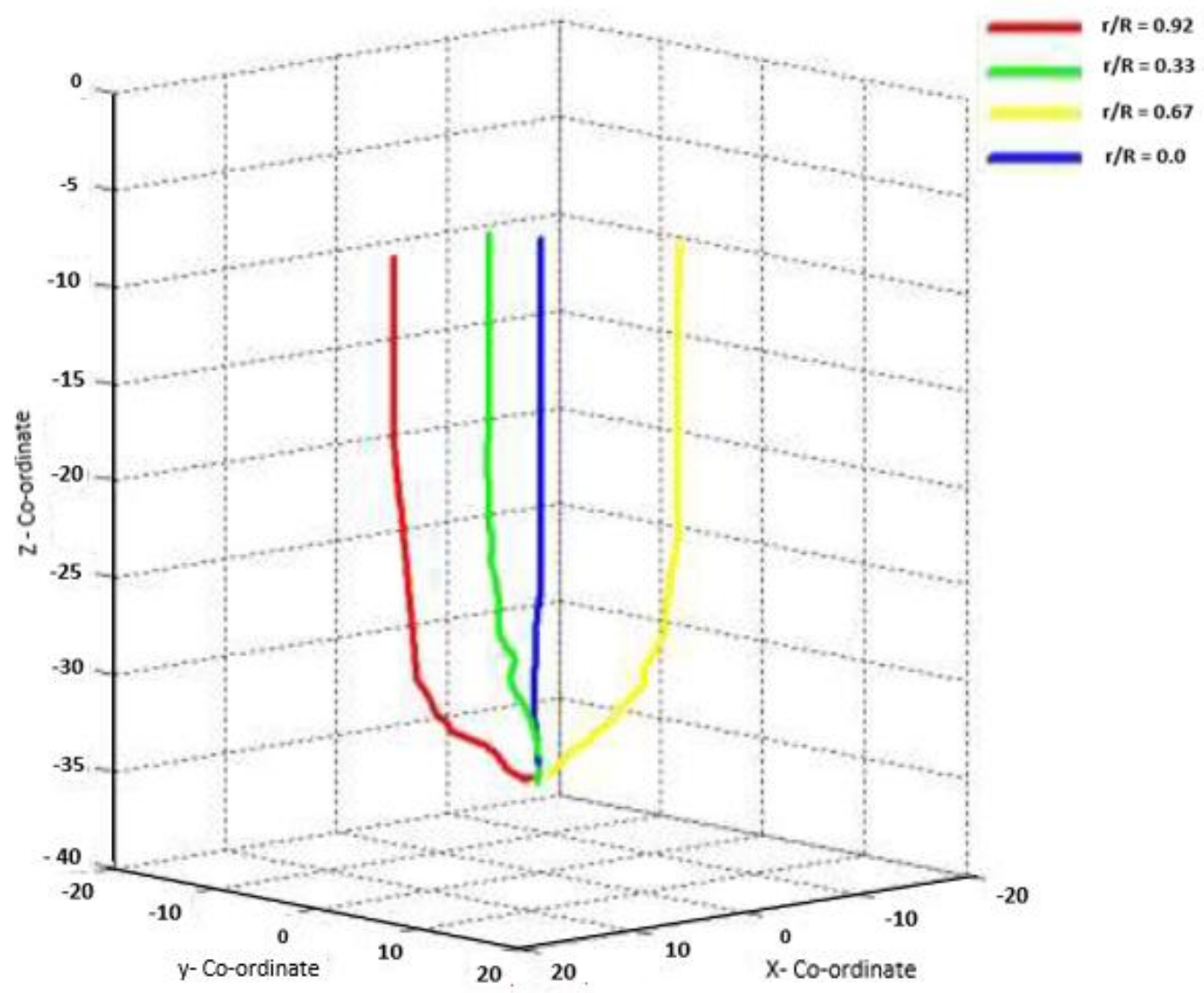

Figure 12 Three-dimensional tracer trajectories obtained using RPT

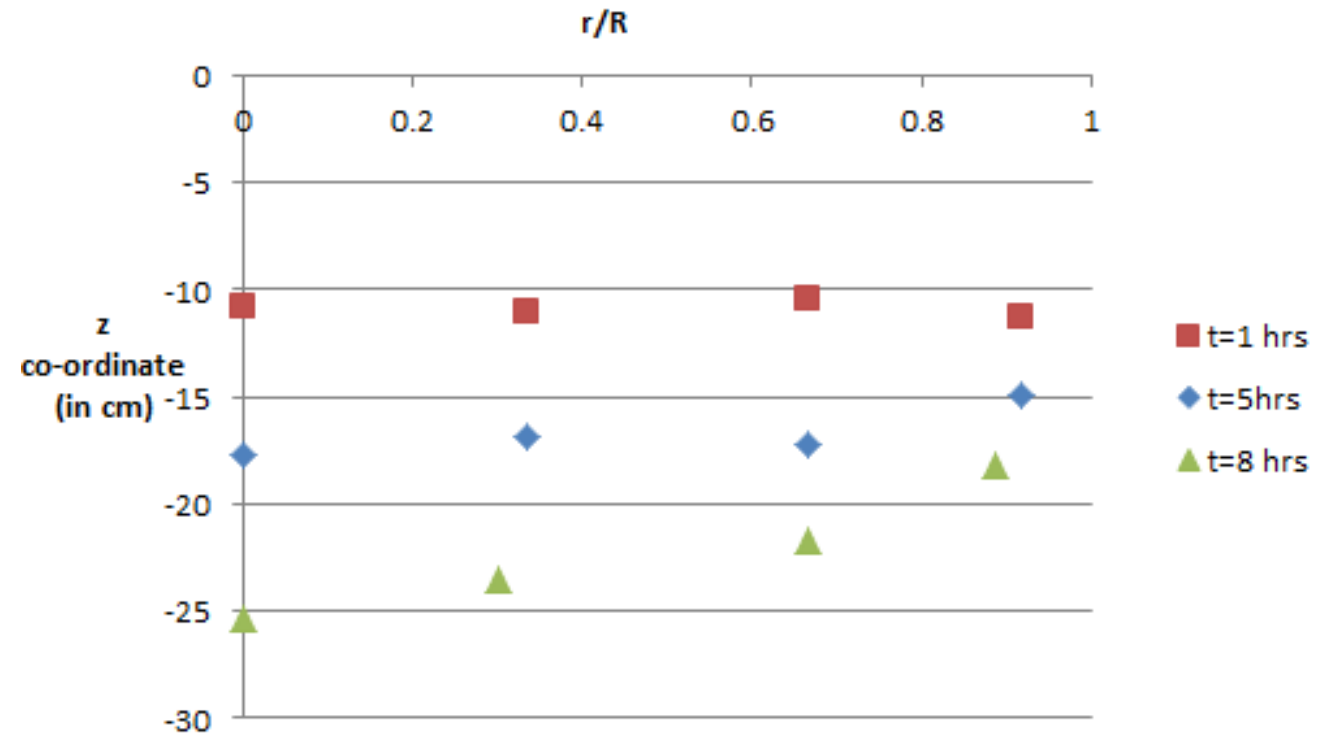

Figure 13 Tracer positions for different initial seeding positions as a function of time. 


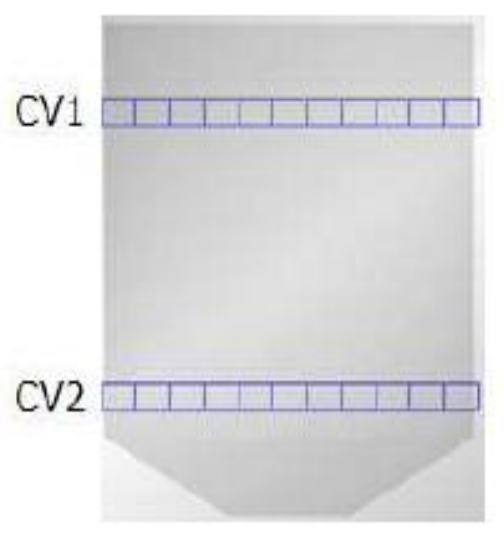

a. Locations of control volume (CV)

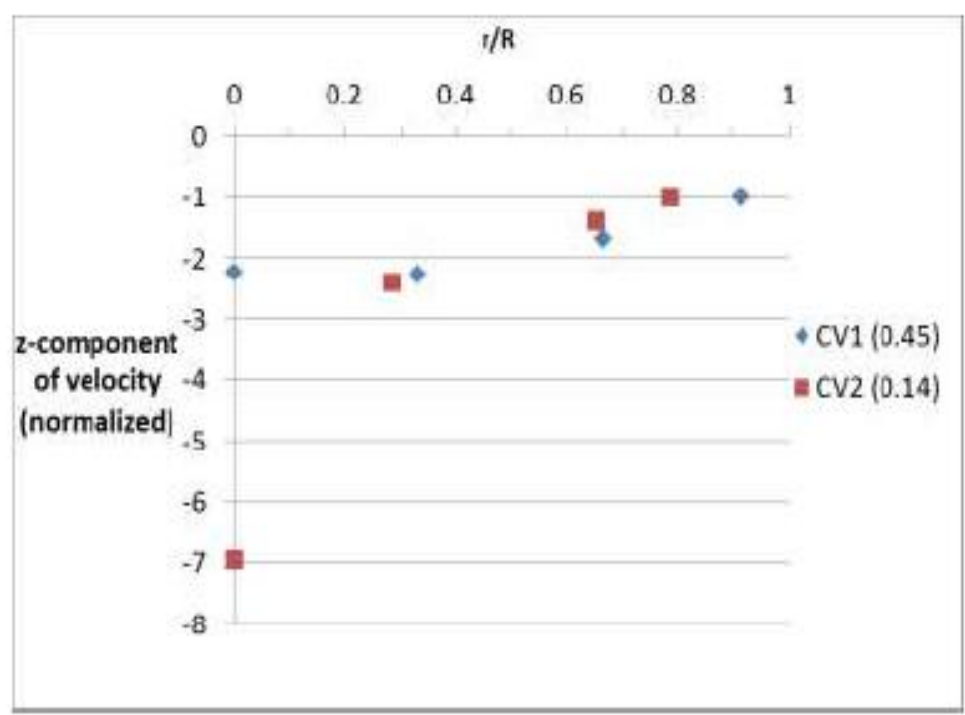

b. Pebbles velocity radial profile (numbers in bracket indicates mass flow index) (MFI) values)

Figure 14 Pebbles velocity radial profile obtained using RPT 


\section{Dr. Muthanna H. Al-Dahhan}

Contact Information: Professor and chairman, department of chemical and biochemical engineering and professor of nuclear engineering, Missouri University of Science and Technology (Missouri S\&T), 110 Bertelsmeyer Hall, 1101 N. State Street, Rolla, MO, United States

Tel: 314-498-9662, 573-341-4416.

Email: aldahhanm@mst.edu

\section{Education and Training}

University of Baghdad, Chemical Engineering

B.S. 1979

Oregon State University, Chemical Engineering

M.S. 1988

(Minor courses in Nuclear Engineering)

Washington University (WU), Chemical Engineering D.Sc. 1993

Research and Professional Experience

Professor and Chairman (Missouri S\&T), Chemical Engineering \&

Jan., 2009-Present

Professor of Nuclear Engineering

Member Energy and Development Center (Missouri S\&T)

Member, Environmental Research Center (Missouri S\&T)

Aug., 2009-Present

Professor (with Tenure), Washington University

Aug., 2009-Present

Co-Director, Chemical Reaction Engineering Laboratory

$2005-2008$

Co-leader NSF Center for Environmental Beneficial Catalysis, University

$2003-2008$

Of Kansas, Lawrence

Associate Professor (with Tenure), Washington University

$2003-2008$

Assistant Professor (Tenure-track), Washington University

$2002-2005$

Associate Director, Chemical Reaction Engineering Laboratory

$1999-2002$

$1994-2003$

Washington University

Assistant Professor, Part-time, (Non-Tenure-track), Washington University

$1994-1999$

Project Manager, Xytel Corporation (pilot plants developer)

$1993-1994$

Process Engineer, R\&D Pilot Plant

$1979-1985$

\section{Publications}

[1] Rahman S. Abdulmohsin and Muthanna Al-Dahhan (2015) Characteristics of Convective Heat Transport in a Packed Pebble-Bed Reactor Using Novel Non-Invasive Heat Transfer Probe, Nuclear Engineering and Design, 284, pp. 143-152 DOI information: 10.1016/j.nucengdes.2014.11.041, 2015.

[2] Kagumba,M.O.;Al-Dahhan M.H.; Impact of Internals Size and Configuration on Bubble Dynamics in Bubble Columns for Alternative Clean Fuels Production Ind. Eng. Chem. Res.2015.

[3] Khairul Anuar Mohd Salleh, Hyoung Koo Lee, Muthanna H. Al-Dahhan (2014), Local liquid velocity measurement in trickle bed reactors (TBRs) using the X-ray digital industrial radiography (DIR) technique, Measurement Science and Technology (Accepted, In Press)

[4] Youssef, A., Hamed, M., Grimes, J., Al-Dahhan, M., and Duduković, M. P., (2013), Hydrodynamics of pilot scale bubble columns: Effect of internals, Ind. \& Eng. Chem. Res., 52 (1) 
[5] Mehul Vesvikar, Muthanna Al-Dahhan, (2012), Development, validation and implementation of multiple radioactive particle tracking (MRPT), Peer Reviewed Proceeding of 6th International Symposium on Process Tomography, Cape Town (South Africa), March 25-28

[6] Abdulmohsin, R., Al-Dahhan, M., (2012), Impact of internals on the heat transfer coefficient in a bubble column, Industrial \& Engineering Chemistry Research, 51 (7), pp. 2874-2881

[7] Wu, C., Al-Dahhan, M., (2012), Heat transfer coefficients in mimicked Fischer-Tropsch slurry bubble columns, Industrial and Engineering Chemistry Research 51 (4), pp. 1543-1548.

[8] Luo, H.-P., Al-Dahhan, M.H., (2011), Verification and validation of CFD simulations for local flow dynamics in a draft tube airlift bioreactor, Chem. Eng. Sci., 66(5), pp.907-923.

[9] Muthanna Al-Dahhan, (2009) Radioisotopes applications in industry: an overview, Atoms for Peace an International Journal, Vol. 2, No. 4, 324-337

[10] Varma R., Bhusarapu S., O’Sullivan J. A., Al-Dahhan M. H., (2008), A comparison of alternating minimization and expectation maximization algorithms for single source gamma ray tomography, Measurement Science and Technology, 19(1), 015506, 14pp.

\section{Synergistic Activities:}

1) Since 1994, I have taught and supervised research on the advancement of transport (hydrodynamics, heat, mass) phenomena engineering methodologies via developing advanced measurement techniques that include non-invasive radioisotopes-based techniques and non-radioisotopes-based sophisticated techniques and modeling and simulating transport-reaction interactions for the proper and efficient design and scale-up of multiphase flow systems for various applications including alternative energy production 2) I have developed and supervised design, scale-up and operations of many multiphase flow pilot plants for fuel and gas processing.

3) I have been working on advancing the knowledge of pebble bed reactors as one of the very high temperature reactors via developing and implementing advanced non-invasive techniques for flow and structure imaging and visualization such as radioactive particle tracking (RPT), gamma ray computed tomography (CT), and gamma ray densitometry (GRD). In addition sophisticated techniques such as gas dynamics tracer and heat transfer probes have been developed and utilized for the related investigations. 4) I have been working on advancing the knowledge and scale up of TRISO particle coating in gas-solid spouted beds using the advanced techniques mentioned above including the development of new sophisticated optical probe that can measure simultaneously solids concentration (holdup), velocity and their time series fluctuations.

5) I worked with a multidisciplinary team at Oak Ridge National Laboratory as a part of DOE grant related to biogas production on developing first of its kind dual source dual energy gamma ray computed tomography (DSCT) and multiple radioactive particle tracking techniques for imaging and visualization of multiphase flow systems which are now located in my laboratory. 